Research Article

\title{
p-Optimality-Based Multiobjective Root System Growth Algorithms for Multiobjective Applications
}

\author{
Rui Liu, ${ }^{1,2}$ Hanning Chen $\mathbb{D}^{,}{ }^{3}$ Lina Song, ${ }^{3}$ and Man Ding ${ }^{4}$ \\ ${ }^{1}$ College of Mathematics, Jilin University, Changchun, China \\ ${ }^{2}$ College of Mathematics, Jilin Normal University, Siping, China \\ ${ }^{3}$ School of Science and Technology, Tianjin Polytechnic University, Tianjin, China \\ ${ }^{4}$ School of Architecture and Art Design, Hebei University of Technology, Tianjin, China \\ Correspondence should be addressed to Hanning Chen; perfect_chn@hotmail.com
}

Received 2 April 2019; Revised 3 June 2019; Accepted 9 July 2019; Published 8 September 2019

Academic Editor: Ricardo Perera

Copyright ( 2019 Rui Liu et al. This is an open access article distributed under the Creative Commons Attribution License, which permits unrestricted use, distribution, and reproduction in any medium, provided the original work is properly cited.

\begin{abstract}
In this paper, a multiobjective root system growth algorithm-based $p$-optimality ( $p$-MORSGA) is proposed. The proposed $p$-MORSGA extended original root system growth algorithm with multiobjective nondomination strategy. To enhance its effect of convergence of solution groups, the $p$-optimality criterion is employed to determine the solutions of last nondominated front into the next generation group. In the evolution process, global general (GG), concerning the margin information and population density, is selected as the suitable optimality criterion of evaluating the performance of solutions. Application of the new p-MORSGA on several multiobjective benchmark functions shows a marked improvement in performance over the modified classical MOEAs with such criterion. Finally, the proposed p-MORSGA is applied to solve two real-world problems, multiobjective portfolio optimization problems (MOPOPs) and multiobjective optimal power flow (OPF) problems. The experimental results demonstrate that $p$-MORSGA is extremely effective for real-world application problems.
\end{abstract}

\section{Introduction}

For multiobjective optimization problems (MOPs), many objectives should be optimized simultaneously under some constraints. Owing to the problems' particularity, researchers prefer to find an approximation of the Pareto optimal set to support the effectiveness of their proposed approaches. At present, MOPs have made some achievements in academia and industry $[1,2]$, but the complexity is still a noticeable puzzle. Since the traditional linear nonlinear programming method cannot effectively solve MOPs, MOEA has become the main method to obtain approximate Pareto frontier (PF). Therefore, it has been widely used in power dispatch [3], logistics system optimization [4], motor design [5], artificial neural network optimization [6], and other applications.

In recent years, a variety of MOEAs have been proposed. All of them can be divided into three classes: (1) nondominant-sorting-based EAs [7], (2) indicator-based EAs
[8], (3) decomposition-based EAs [9]. All these improved algorithms have the advantage of competitiveness.

In the existing literature [10], root system growth algorithm (RSGA) is presented to solve single-objective problems. The root system growth algorithm is inspired by natural plant root system. Plant root system is characterized by the ability to adapt to the changing diversity of the environment [11-13]. The goal of this paper is to use RSGA to solve multiobjective problems such as MOEAs. As conventional evolutionary algorithms for MOPs, RSGA should design multiobjective mechanism to balance its diversity and convergence.

The constructed and updated Pareto nondominant set can be approximately represented by the real optimal frontier. This method will obtain an acceptable result when the advantage relationship in the target space is obvious, while it will show poor performance when the advantage relationship is weak or when the multimodal and deceptive problems are optimal in isolation [14]. 
In view of this situation, Pareto dominance processing technology is divided into the following types:

(i) Randomized schemes, such as roulette selection [15], result in inefficiencies and poor solution caliber.

(ii) Pareto-based relaxed domination methods, such as $\varepsilon$-domination [16], $r$-domination [17], grid-based method [18], etc.

(iii) Reference-point-based dominance methods [19, 20] adjusting solutions according to reference points.

(iv) In the $k$-optimality used in literature [21], the dominant solution may not perform well on some specific targets, which is acceptable to decision makers. The number of targets was previously specified by adjusting the $k$ value.

(v) Optimality criterion in [22] is adopted to search the optimal spatial position meeting certain predetermined criteria, and this hypothesis is assumed to be the corresponding optimal result of the problem.

(vi) Average ranking (AR) [23], preference order ranking (PO) [24], global general (GG) [25], and so on.

In this paper, $p$-optimality criteria combining RSGA are proposed to solve MOP. $p$-Optimality criterion is applied to selection operators in the evolutionary process. Such criteria are helpful to distinguish the viable solutions among solutions at the same nondominated level. Therefore, it is possible for them to converge better on feasible solutions at a later stage of evolution. Meanwhile, global general (GG) criterion is selected as the suitable optimality criterion of evaluating the performance of solutions. Global general, a ranking-based dominant mechanism, uses the margin information of solutions with concerning population density (Euclidean distance among the solutions).

With the test against several multiobjective benchmark functions, the results demonstrate that the proposed $p$-optimality-based multiobjective root system growth algorithm ( $p$-MORSGA) outperforms NSGA-II, MOEA/D, MOPSO, rNSGA-II, and RPDNSGA-II (classical multiobjective algorithms with $p$-optimality criteria) on convergence metric and diversity metric. In order to test the effect of real-world multiobjective applications with $p$-MORSGA, multiobjective portfolio optimization problems (MOPOPs) and multiobjective optimal power flow (OPF) are employed.

In [26], the proposed multiobjective portfolio selection model has been transformed into a single-objective programming model using fuzzy normalization and uniform design method. The model is then applied to monthly data of top 50 companies of Tehran Stock Exchange Market in 2013. The experiment concluded that IWO algorithm has better solving time than PSO algorithm. Mehlawat uses the return, risk, and liquidity model in [27] to better solve the problem of fuzzy combination parameters in financial markets. Jiang proposed a general multiobjective CVaR model in time interval for loan portfolios in [28]. The results prove that this model can better deal with the actual financial risk problem.
Yuan et al. [29] proposed an improved strength Pareto evolutionary algorithm and applied it to the OPF problem. The experiment results show that the improved algorithm has obvious performance. In [30], the paper presents an enhanced self-adaptive differential evolution with mixed crossover (ESDE-MC) algorithm to solve the multiobjective OPF problems with conflicting objectives that reflect the minimization of total production cost, emission pollution, L-index, and active power loss. Teeparthi and Vinod Kumar [31] proposed a new low level with teamwork heterogeneous hybrid particle swarm optimization and artificial physics optimization (HPSO-APO) algorithm. The algorithm balances global and local search capabilities. Hence, the proposed hybrid method can be used for the large interconnected power system to solve MO-SCOPF problem with integration of wind and thermal generators.

Portfolio management refers to the investment manager's diversification management of assets according to asset selection theory and portfolio theory to achieve the purpose of diversifying risks and improving efficiency. The application of EAs in solving MOPOP has attracted wide attention, yet their method is usually to convert MOPOP into single-objective POP by weighted sum. Therefore, OPF problem is to schedule the promised generator set to meet system load requirements with minimal costs under some constraints. OPF problem is a multiobjective nonlinear constrained optimization problem of competition. The experimental results show that $p$-MORSGA outperforms MOEAs in terms of accuracy and computation.

The remainder of this paper is organized as follows. Section 2 introduces related works of this paper, $p$-optimality criterion and global general criterion. Section 3 describes original root system growth algorithm. Section 4 describes a novel optimization model called $p$-MORSGA. In Section 5 provides the extensive experimental results and discussion. In Section 6, p-MORSGA for MOPOP and OPF is presented. Finally, Section 6 gives the conclusion.

\section{Related Works}

2.1.p-Optimality Criterion. p-Optimality criteria $[32,33]$ are defined as follows:

$$
P_{i}(v)=1-\frac{S_{i}(v)+1}{\left|K^{\prime}\right|},
$$

where $S_{i}(v)$ is the rank according to the objective function $f_{i}$ by means of the quick-sort algorithm and $|K|$ is the size of feasible solutions.

Therefore, the goal is to search for a vector $v^{*} \subseteq K^{\prime}$ that maximizes $f_{i}(v)=k^{-1} \cdot \sum_{i=1}^{k} P_{i}(v)$ or $f(v)=\sum_{i=1}^{k} p_{i}(v)$ :

$$
\begin{aligned}
\max f(v) & =\max \left\{k-\sum_{i=1}^{k} P_{i}(v)\right\}=\max \left\{\sum_{i=1}^{k}\left(1-P_{i}(v)\right)\right\} \\
& =\max \left\{\sum_{i=1}^{k}\left|1-P_{i}(v)\right|\right\} .
\end{aligned}
$$

Consider the following functions: 


$$
p-\text { function }(v)=\left(\sum_{i=1}^{k}\left(1-P_{i}(v)\right)^{p}\right)^{1 / p}
$$

where $p>0$, and $p_{i}(a)$ is calculated as equation (1).

Such criterion promises the most feasible solution in the same nondominant rank.

\subsection{Global General Criterion}

Definition 1 (global margin, GM). GM is defined as the sum of all the individual target values of the difference:

$$
\mathrm{GM}\left(X_{i}\right) \triangleq \sum_{X_{i} \neq X_{j}} \max \left(\left(\prod_{m=1}^{M} f_{m}\left(X_{i}\right)-\prod_{m=1}^{M} f_{m}\left(X_{j}\right)\right), 0\right) \text {, }
$$

where $M$ is set to the number of objectives and $X_{i}$ and $X_{j}$ are two different solutions.

According to Pareto dominance, the smaller $\mathrm{GM}\left(X_{i}\right)$, the more $X_{i}$ will dominate.

The framework combines each goal with the information of all individuals to obtain ranking values. By comparing with the solution pairs, the sum of the good or bad parts of the solution pairs is calculated, as shown in Figure 1.

Definition 2 (global density, GD).

$$
\mathrm{GD}\left(X_{i}\right)=\sum_{j=1, i \neq j}^{\text {pop }} d_{i, j}
$$

where $\mathrm{GM}\left(X_{i}\right)$ denotes the global density of the particle $X_{i}$ and $d_{i, j}$ being the Euclidean distance.

Definition 3 (global general, GG).

$$
\mathrm{GG}\left(X_{i}\right) \triangleq \frac{\mathrm{GM}\left(X_{i}\right)}{\mathrm{GD}\left(X_{i}\right)}
$$

where $\mathrm{GG}\left(X_{i}\right)$ represents the overall ranking of $X_{i}$. The smaller $\mathrm{GG}\left(X_{i}\right)$ indicates that the decent GD of $X_{i}$ has a better distribution.

\section{Multiobjective Root System Growth Algorithm}

This section describes the $p$-optimality-based multiobjective root system growth algorithm ( $p$-MORSGA).

The root system is defined as a group of tips, which are expressed as

$$
R S=\left\{\theta_{i}^{t} \mid i=1,2, \ldots, P^{t} ; \quad t=1,2, \ldots, T\right\},
$$

where $\theta_{i}^{t}=\left\langle x_{i}^{t}, f_{i}^{t}, n_{i}^{t}, \alpha_{i}^{t}\right\rangle$ denotes a single root tip; $P^{t}$ denotes the root tips' number; $T$ is the last time; and $\theta_{i}^{t}$ has its own position $x_{i}^{t}$, fitness $f_{i}^{t}$, nutrient $n_{i}^{t}$, and the auxin $\alpha_{i}^{t}$.

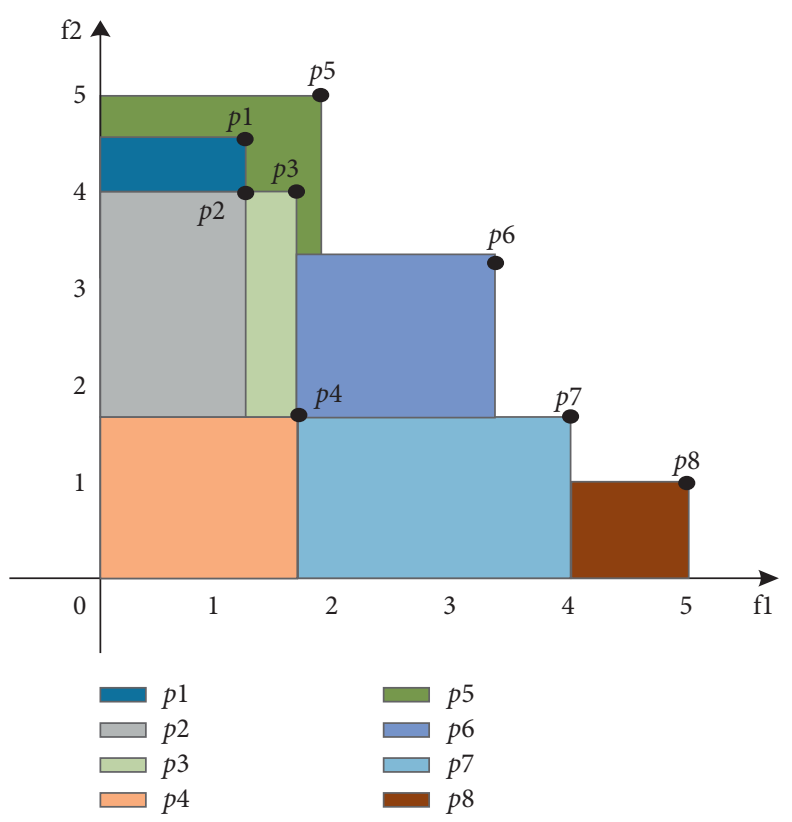

FIgURE 1: A sketch map on global margin ranking.

3.1. Auxin Concentration. In initialization, $P^{0}$ are generated in $D$-dimensional space. The root tips $i$ will forage for nutrients, which can be updated in the following ways:

$$
n_{i}^{t+1}= \begin{cases}n_{i}^{t}+1, & \text { if } \mathrm{GG}_{i}^{t+1}<\mathrm{GG}_{i}^{t}, \\ n_{i}^{t}-1, & \text { else, }\end{cases}
$$

where GG is the global general metric introduced in Section 2 .

In the initial stage, the nutritional value of each root tip is set as 0 . During root growth, if its new position is better than the last one, the tip will receive nutrients from the environment. Inversely, the tips will lose nutrients and the nutrient content will be decreased by one.

Then, the auxin concentration of auxin $\alpha_{i}^{t}$ is manipulated according to the following equation, which combined with the health status:

$$
\alpha_{i}^{t}=\frac{\mathrm{GG}_{i}^{t}-\mathrm{GG}_{\mathrm{worst}}^{t}}{\mathrm{GG}_{\text {best }}^{t}-\mathrm{GG}_{\text {worst }}^{t}}
$$

where $J_{\text {worst }}$ and $J_{\text {best }}$ are the worst and most suitable place and GG is the global general metric.

All root taps are classified according to the auxin concentration values. The stronger tip has a higher probability of becoming the main root. The size of main roots is limited as follows:

$$
S_{m}^{t}=P^{t} \times Q
$$

where $S_{m}^{t}$ is the number of the main root group, $P^{t}$ is the total number of root tips, and $Q$ is the selection factor. The other $S_{l}^{t}=P^{t}-S_{m}^{t}$ root tips are considered as lateral roots.

3.2. Root Branching. The threshold $B_{G}$ is to determine whether main root can be regarded as branch. If $\alpha_{i}^{t}$ is greater than $B_{G}$, the root is considered as the branch root.

The number of branching $w_{i}$ of $\theta_{i}^{t}$ is decided by the following formula: 
(1) 1: Initialization.

(2) Iteration $=0$;

(3) Initialize $P^{0}$, the positions of root tip group;

(4) Evaluate the fitness of the group;

(5) Evaluate the global general (GG) of each solution by equations (4)-(6)

(6) 2: Do while (unsatisfactory conditions)

(7) 3: Auxin concentration calculation

(8) Compute the auxin concentration values of the root tip group by equation (9);

(9) Sort the root tip group in order of descending accumulated auxin concentration;

(10) Divide the root tip group into two subgroups, i.e. the main root group and the lateral root group of equation (10).

(11) 4: Root branching

(12) Determine the branch number of each branching root tip by equation (11);

(13) 5: Main roots growing

(14) Sort the main root subgroup in order of increasing cumulative auxin concentration;

(15) The first half main roots are hydrotropic according to equation (12);

(16) 6: Lateral roots growing

(17) All the lateral root tips perform random walk operation according to equation (13);

(18) 7: Dead roots eliminating

(19) Remove the dead root tips (auxin concentration 0) from the root tip group;

(20) 8: Generate offspring group

(21) Gather the new generated solution as offspring group $O^{t}$;

(22) 9: Determine the solutions of the next iteration

(23) Combine parent group and offspring group $Q^{t}=P^{t} \cup Q^{t}$

(24) Non-dominated sort on Pareto dominance $\left\{F_{1}, F_{2}, \ldots\right\}$

(25) Add nondominated front $F_{i}$ into the next group $P^{t+1}$ one by one until $\left|P^{t+1} \cup F_{i}\right| \geq\left|P^{0}\right|$

(26) Add the solutions of last nondominated front $F_{i}$ with the largest $p$-optimality criterion one by one until $\left|P^{t+1}\right|=\left|P^{0}\right|$.

(27) 8: Memorize the best solution to date

(28) $\quad$ Iteration $=$ iteration +1 ;

(29) End while

(30) 9: Output the best solution achieved.

Algorithm 1: Pseudocode of $p$-MORSGA.

$$
S_{i}^{t}=\left\lceil\operatorname{Rand} * \alpha_{i}^{t}\left(S_{\max }-S_{\min }\right)+S_{\min }\right\rceil,
$$

where $S_{\max }$ and $S_{\min }$ are the maximum and minimum limits.

The new branching tips foraging in the new regions will grow.

The fitness $f$ is the result of applying the following functions to each successive group of three components:

$$
x_{j}^{t+1}=x_{i}^{t}+\text { Rand } * l_{\max },
$$

where $l_{\max }$ is the maximum of root elongation length.

3.3. Tropisms. Trajectory of the root is influenced by different tropisms. In the RSGA model, two typical orientations are realized, i.e., hydrotropism and gravitropism.

Half of the main roots will grow to the optimum position, and the water content in the roots will be the largest, which is given by the following formula:

$$
x_{i}^{t+1}=x_{i}^{t}+\text { Rand } *\left(x_{\text {best }}^{t}-x_{i}^{t}\right),
$$

where $i \subseteq\left[1,\left(S_{m}^{t} / 2\right)\right]$ and $x_{\text {best }}^{t}$ is the best position in the root tip group.

3.4. Root Tip Death. Lower auxin concentration indicates that the root tip does not acquire as much nutrients as possible during its foraging and therefore is not active and continuous growing is not possible.
3.5. Pseudocode of p-MORSGA. p-MORSGA algorithm code and flow chart are listed in Algorithm 1.

The algorithm can be divided into six parts. The first part is to initialize the population and calculate the fitness and GG values. The second part is to calculate the auxin concentration values according to the content of the chapter II and then sort them to divide the main root group and the lateral root group. The third and fourth parts are the growth of the main root and the lateral root. Elimination of dead roots is the fifth part. We remove the dead root tips (auxin concentration 0 ) from the root tip group. The sixth part is to determine the solution of the next iteration: first combine parent group and offspring group $Q^{t}=P^{t} \cup Q^{t}$, secondly, perform nondominated sorting, and get $\left\{F_{1}, F_{2}, \ldots\right\}$, in the critical layer $F_{i}$, where $F_{i}$ meets $F_{1} \cup F_{2} \cup \cdots \cup F_{i}-1<P^{t}+1<F_{1} \cup \cdots \cup F_{i}-1 \cup F_{i}$. Then, the $p$-optimality will be used to sort the individuals in the critical layer, where the smaller the $p$-function is, the better the individual is. Finally, select the smallest $\left|P^{0}\right|-\left|F_{1} \cup F_{2} \cup \cdots \cup F_{i}-1\right|$ individuals to make the number of $P^{t+1}$ equal to the number of $P^{0}$.

\section{Tests}

4.1. Experimental Setting. In the two-objective test experiment, population size is set as 200, and the number of function evaluations (FEs) is set as 40000. In the threeobjective test experiment, population size is set as 300, and 
TABLE 1: Test results of two objective functions.

\begin{tabular}{|c|c|c|c|c|c|c|c|c|}
\hline & Problem & & p-MORSGA & NSGA-II & MOEA/D & MOPSO & rNSGA-II & RPDNSGA-II \\
\hline \multirow{8}{*}{ ZDT1 } & Converge & Avg & $8.2473 e-1$ & $9.8750 e-1$ & $8.3829 e-1$ & $1.0000 e+0$ & $9.9750 e-1$ & $9.9477 e-1$ \\
\hline & Metric & Std & $8.23 e-3$ & $9.50 e-3$ & $1.02 e-1$ & $0.00 e+0$ & $4.86 e-3$ & $5.15 e-3$ \\
\hline & Diversity & Avg & $2.2842 e-3$ & $2.3791 e-3$ & $2.6362 e-3$ & $2.0794 e+1$ & $2.9883 e-1$ & $4.9906 e-3$ \\
\hline & Metric & Std & $6.88 e-5$ & $6.99 e-5$ & $8.43 e-4$ & $8.36 e+0$ & $6.08 e-3$ & $5.35 e-4$ \\
\hline & IGD & Avg & $2.2842 e-3$ & $2.3791 e-3$ & $2.6362 e-3$ & $2.0510 e+1$ & $2.9883 e-1$ & $4.9906 e-3$ \\
\hline & Metric & Std & $6.78 e-5$ & $6.99 e-5$ & $8.43 e-4$ & $8.48 e+0$ & $6.08 e-3$ & $5.35 e-4$ \\
\hline & $\mathrm{HV}$ & Avg & $7.2140 e-1$ & $7.2154 e-1$ & $7.2155 e-1$ & $1.0000 e+0$ & $4.1705 e-1$ & $7.1954 e-1$ \\
\hline & Metric & Std & $1.88 e-3$ & $1.44 \mathrm{e}-4$ & $1.07 e-3$ & $0.00 \mathrm{e}+0$ & $5.36 e-3$ & $4.90 \mathrm{e}-4$ \\
\hline \multirow{8}{*}{ ZDT2 } & Converge & Avg & $3.5233 e-1$ & $1.0000 e+0$ & $4.5020 e-1$ & $1.0000 e+0$ & $9.9696 e-1$ & $9.8232 e-1$ \\
\hline & Metric & Std & $1.89 \mathrm{e}-1$ & $0.00+0$ & $2.69 e-1$ & $0.00 e+0$ & $4.85 e-3$ & $3.46 e-2$ \\
\hline & Diversity & Avg & $2.3924 e-3$ & $2.9150 e-3$ & $2.1365 e-3$ & $3.0864 e+1$ & $3.3474 e-1$ & $1.5993 e-2$ \\
\hline & Metric & Std & $4.93 e-4$ & $1.57 e-3$ & $4.70 e-4$ & $8.04 e+0$ & $5.59 e-3$ & $2.65 e-2$ \\
\hline & IGD & Avg & $2.3924 e-3$ & $2.9150 e-3$ & $2.1365 e-3$ & $3.0864 e+1$ & $3.3474 e-1$ & $1.5993 e-2$ \\
\hline & Metric & Std & $2.43 e-4$ & $1.57 e-3$ & $4.70 e-4$ & $8.04 e+0$ & $5.59 e-3$ & $2.65 e-2$ \\
\hline & $\mathrm{HV}$ & Avg & $4.6422 \mathrm{e}-1$ & $4.4460 e-1$ & $4.4628 e-1$ & $1.0000 e+0$ & $1.8738 e-1$ & $4.2840 e-1$ \\
\hline & Metric & Std & $1.89 \mathrm{e}-3$ & $4.58 e-3$ & $1.66 e-3$ & $0.00 e+0$ & $3.13 e-3$ & $3.34 e-2$ \\
\hline \multirow{8}{*}{ ZDT3 } & Converge & Avg & $1.0342 \mathrm{e}-2$ & $8.7650 e-1$ & $2.0135 e-1$ & $1.0000 e+0$ & $7.8232 e-1$ & $8.1864 e-1$ \\
\hline & Metric & Std & $3.24 \mathrm{e}-2$ & $6.57 e-2$ & $1.08 e-1$ & $0.00 e+0$ & $1.13 e-1$ & $1.05 e-1$ \\
\hline & Diversity & Avg & $1.2395 e-2$ & $4.4731 e-2$ & $1.1309 \mathrm{e}-2$ & $1.9225 e+1$ & $5.2856 e-1$ & $4.2074 e-2$ \\
\hline & Metric & Std & $3.43 e-2$ & $8.16 e-2$ & $1.24 \mathrm{e}-2$ & $6.79 e+0$ & $7.40 e-2$ & $4.90 e-2$ \\
\hline & IGD & Avg & $1.2374 e-2$ & $4.4731 e-2$ & $1.1309 \mathrm{e}-2$ & $1.8946 e+1$ & $5.2856 e-1$ & $4.2074 e-2$ \\
\hline & Metric & Std & $1.02 e-2$ & $8.16 e-2$ & $1.24 \mathrm{e}-2$ & $6.63 e+0$ & $7.40 e-2$ & $4.90 e-2$ \\
\hline & $\mathrm{HV}$ & Avg & $6.7935 e-2$ & $6.3195 e-1$ & $6.0250 e-1-$ & $1.0000 e+0$ & $5.3392 e-1$ & $6.5979 e-1$ \\
\hline & Metric & Std & $1.33 e-3$ & $6.82 e-2$ & $4.07 e-2$ & $0.00 e+0$ & $1.21 e-2$ & $8.87 e-2$ \\
\hline \multirow{8}{*}{ ZDT4 } & Converge & Avg & $8.3583 e-1$ & $9.9500 e-1$ & $1.0000 e+0-$ & $1.0000 e+0$ & $1.0000 e+0$ & $9.6527 e-1$ \\
\hline & Metric & Std & $3.92 \mathrm{e}-3$ & $4.08 e-3$ & $0.00 e+0$ & $0.00 e+0$ & $0.00 e+0$ & $4.01 e-2$ \\
\hline & Diversity & Avg & $2.9400 \mathrm{e}-3$ & $3.0137 e-3$ & $4.3406 e-3$ & $1.4861 e+1$ & $2.8996 e-1$ & $1.1594 e-1$ \\
\hline & Metric & Std & $5.89 e-4$ & $5.73 e-4$ & $1.50 e-3$ & $5.97 e+0$ & $3.95 e-3$ & $3.16 e-2$ \\
\hline & IGD & Avg & $2.9400 e-3$ & $3.0137 e-3$ & $4.3406 e-3$ & $1.4453 e+1$ & $2.8996 e-1$ & $1.1594 e-1$ \\
\hline & Metric & Std & $5.89 e-4$ & $5.73 e-4$ & $1.50 e-3$ & $5.84 e+0$ & $3.95 e-3$ & $3.16 e-2$ \\
\hline & $\mathrm{HV}$ & Avg & $7.3248 \mathrm{e}-1$ & $7.2019 e-1$ & $7.1808 e-1-$ & $1.0000 e+0$ & $4.2437 e-1$ & $6.1428 e-1$ \\
\hline & Metric & Std & $2.55 e-3$ & $1.01 e-3$ & $2.12 e-3$ & $0.00 e+0$ & $3.32 e-3$ & $3.55 e-2$ \\
\hline \multirow{8}{*}{ ZDT6 } & Converge & Avg & $3.0324 \mathrm{e}-1$ & $9.5450 e-1$ & $9.1000 e-1$ & $2.0450 e-1$ & $9.7400 e-1$ & $9.9829 e-1$ \\
\hline & Metric & Std & $1.34 \mathrm{e}-1$ & $9.55 e-2$ & $1.34 e-1$ & $3.96 e-1$ & $8.22 e-2$ & $3.86 e-3$ \\
\hline & Diversity & Avg & $1.0348 e-3$ & $1.8732 e-3$ & $1.6607 e-3$ & $9.5418 e-1$ & $2.2503 e-1$ & $5.7035 e-3$ \\
\hline & Metric & Std & $4.66 e-5$ & $9.17 e-5$ & $7.58 e-5$ & $2.96 e+0$ & $5.43 e-3$ & $2.87 e-4$ \\
\hline & IGD & Avg & $1.0348 e-3$ & $1.8732 e-3$ & $1.6607 e-3$ & $9.3956 e-1$ & $2.2503 e-1$ & $5.7035 e-3$ \\
\hline & Metric & Std & $4.66 e-5$ & $9.17 e-5$ & $7.58 e-5$ & $2.96 e+0$ & $5.43 e-3$ & $2.87 e-4$ \\
\hline & $\mathrm{HV}$ & Avg & $2.9843 e-1$ & $3.8983 e-1$ & $3.8985 e-1$ & $3.4808 e-1$ & $2.0532 e-1$ & $3.8554 e-1$ \\
\hline & Metric & Std & $4.32 e-3$ & $2.98 e-4$ & $2.34 \mathrm{e}-4$ & $1.22 e-1$ & $3.40 e-3$ & $3.95 e-4$ \\
\hline
\end{tabular}

the number of function evaluations (FEs) is set as 90000. The number of independent runs of the experiment is 10 times. For $p$-MORSGA, NSGA-II, MOEA/D, MOPSO, rNSGA-II, and RPDNSGA-II, parameter settings are the same as the ones in $[8,17,34-37]$, respectively. For $p$-MORSGA, the whole swarm has 200 individuals, and parameter settings are the same as the ones in [32].

\subsection{Results and Discussion}

4.2.1. Two-Objective Functions. Table 1 and Figures 2-6 demonstrate the results of $p$-MORSGA and MOEAs against ZDT series.

In Table 1, four metrics are used to compare the performance of the algorithms. In order to facilitate reading, the best test results are marked in bold. In general, $p$-MORSGA performs best, followed by the MOEA/D.
Figures 2-6 show that MOEAs produce poor results on these test functions and they are almost impossible to achieve true PF, while $p$-MORSGA has great potential to approach true PF. Obviously, for the uniformity of the final solution, $p$-MORSGA has better results for the ZDT series test function. At the same time, MOEA/D has the secondbest effect, and rNSGA and RPDNSGA-II perform poorly. In general, $p$-MORSGA is superior in terms of uniformity and coverage, followed by MOEA/D; NAGA-II is not uniform, rNSGA-II and RPDNSGA-II are not ideal, and MOPSO algorithm has the worst performance except ZDT3.

4.2.2. Three-Objective Functions. Table 2 and Figures 7-10 demonstrate the results of $p$-MORSGA and MOEAs against DTLZ1, DTLZ2, and DTLZ3. The true Pareto frontier is shown in Figure 7 for three-objective functions. The results 

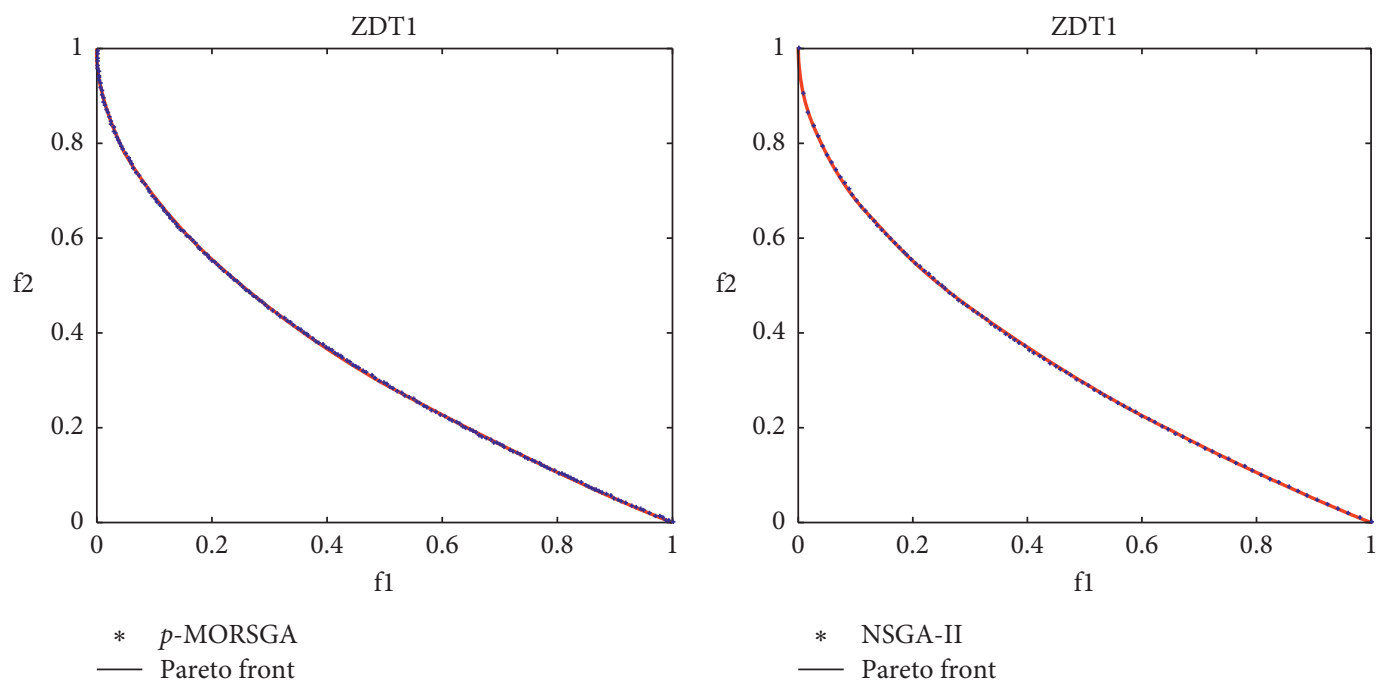

(a)

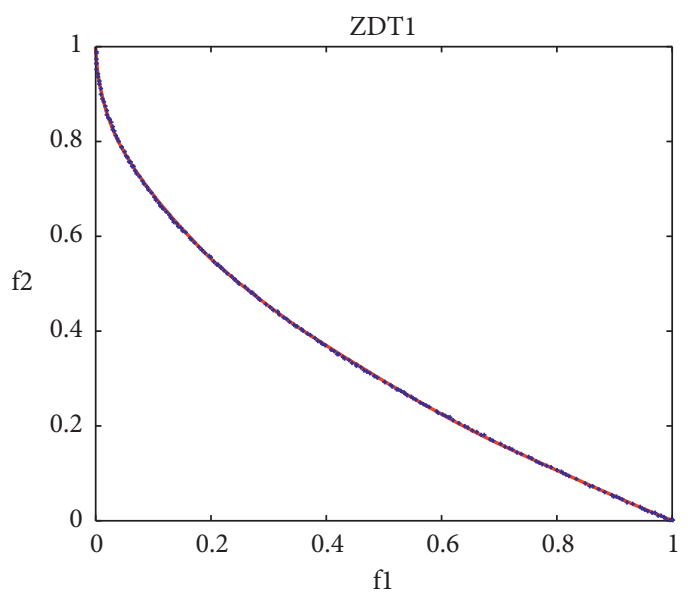

(b)

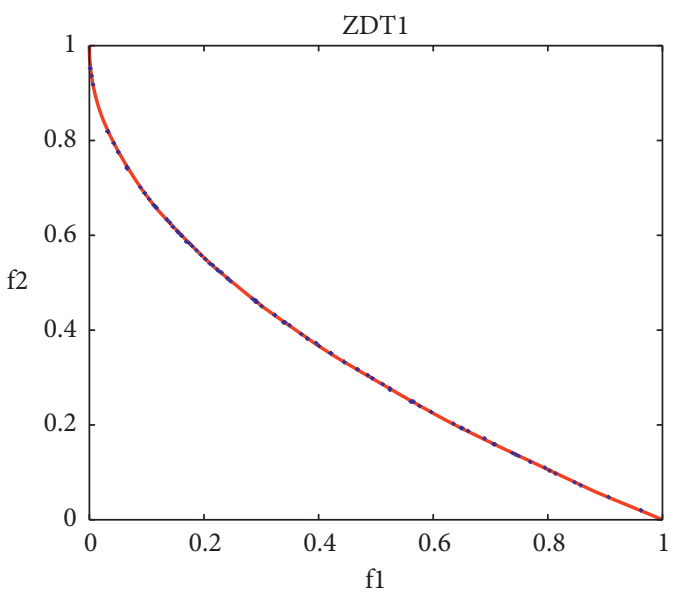

* MOEA/D

— Pareto front

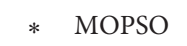

— Pareto front

(c)

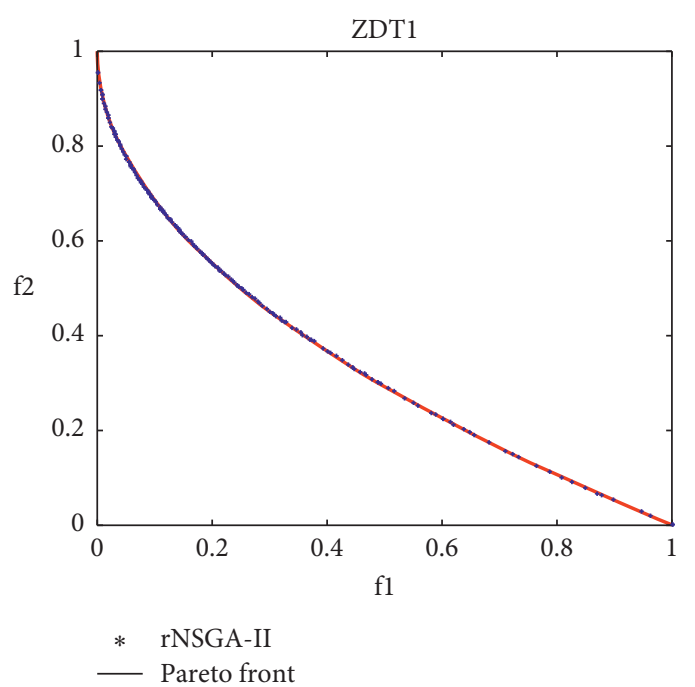

(e)

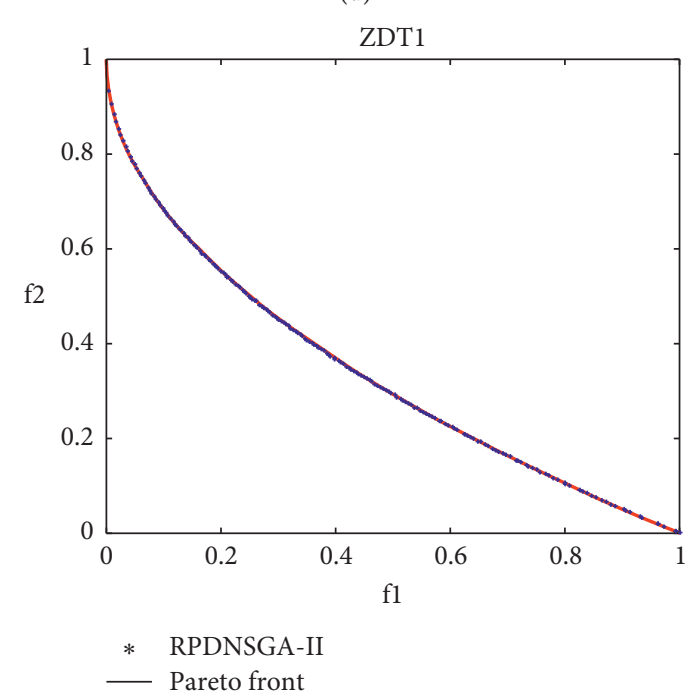

(f)

FIgure 2: Obtained results on ZDT1: (a) PF obtained by p-MORSGA on ZDT1; (b) PF obtained by NSGA on ZDT1; (c) PF obtained by MOEA/D on ZDT1; (d) PF obtained by MOPSO on ZDT1; (e) PF obtained by rNSGA-II on ZDT1; (f) PF obtained by RPDNSGA-II on ZDT1. 

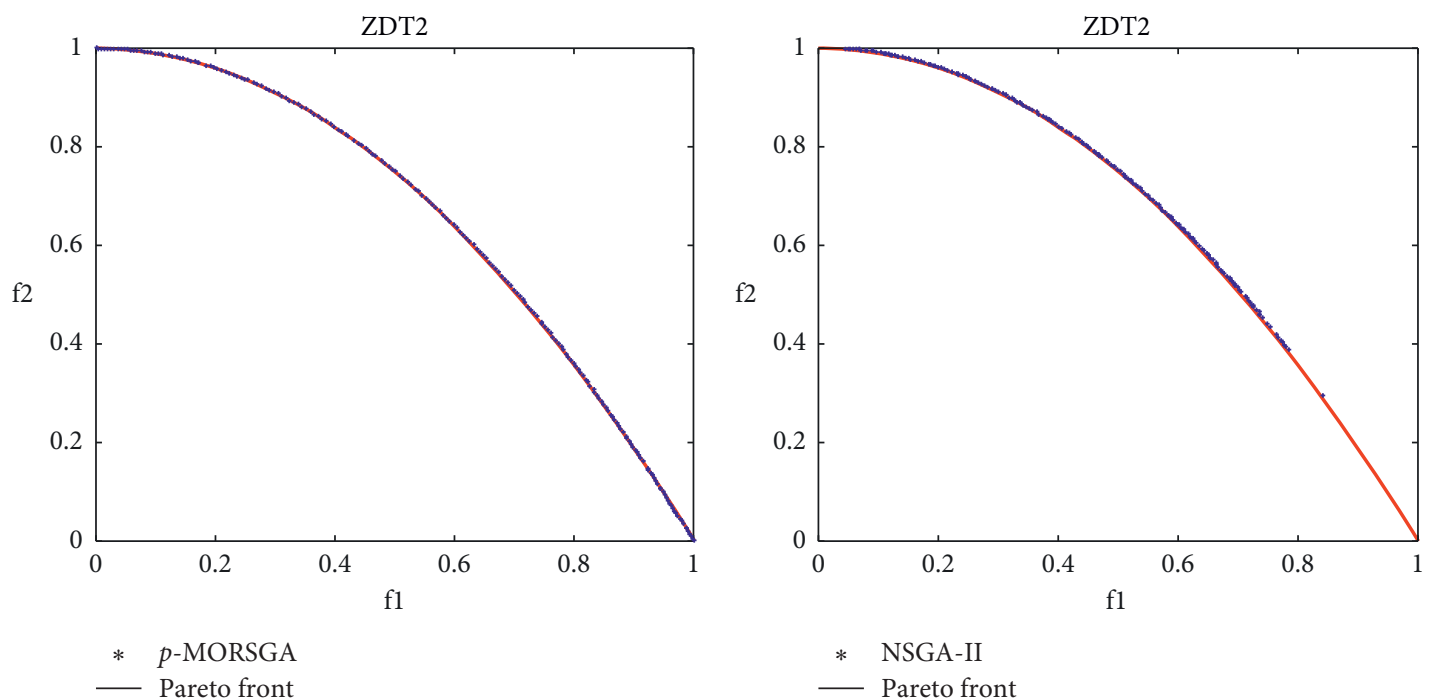

(a)

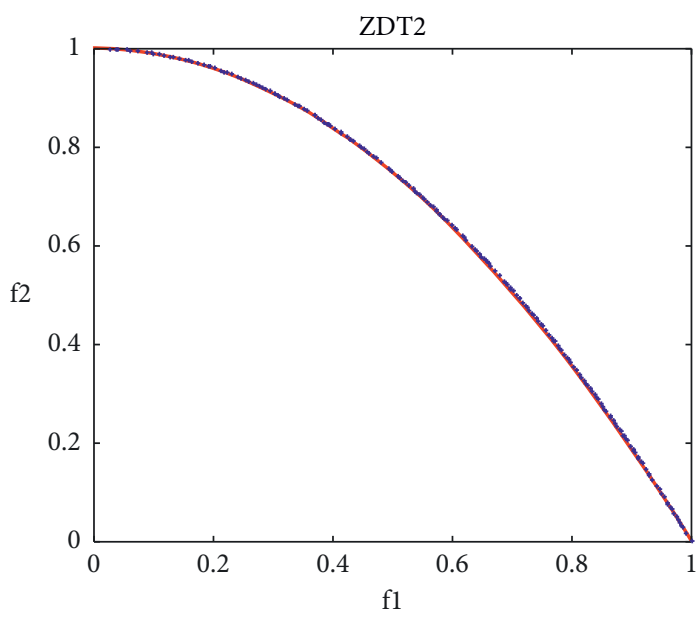

(b)

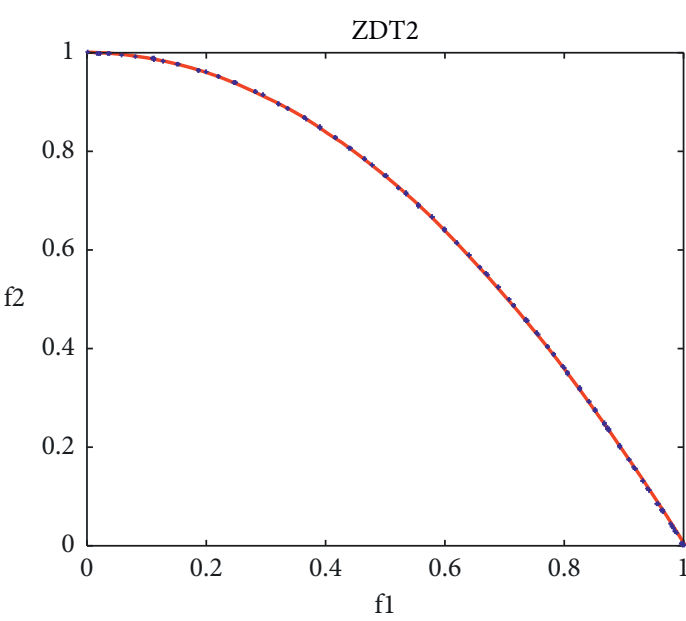

* MOEA/D

* MOPSO

— Pareto front

- Pareto front

(c)

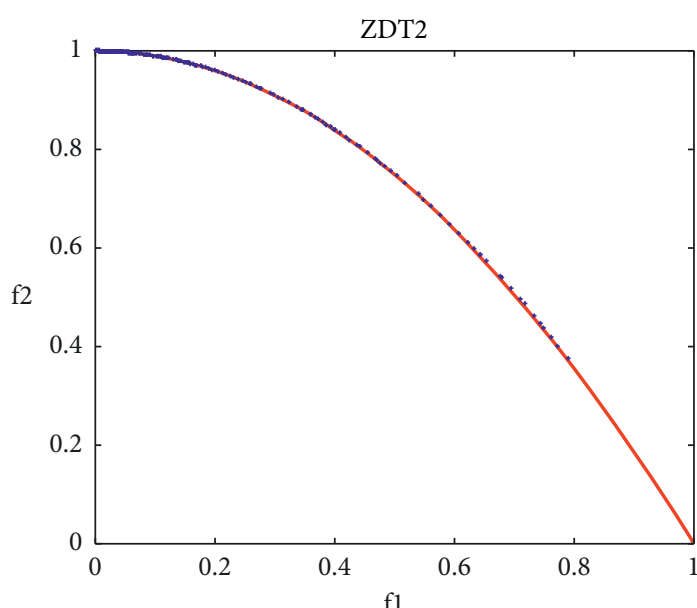

(d)

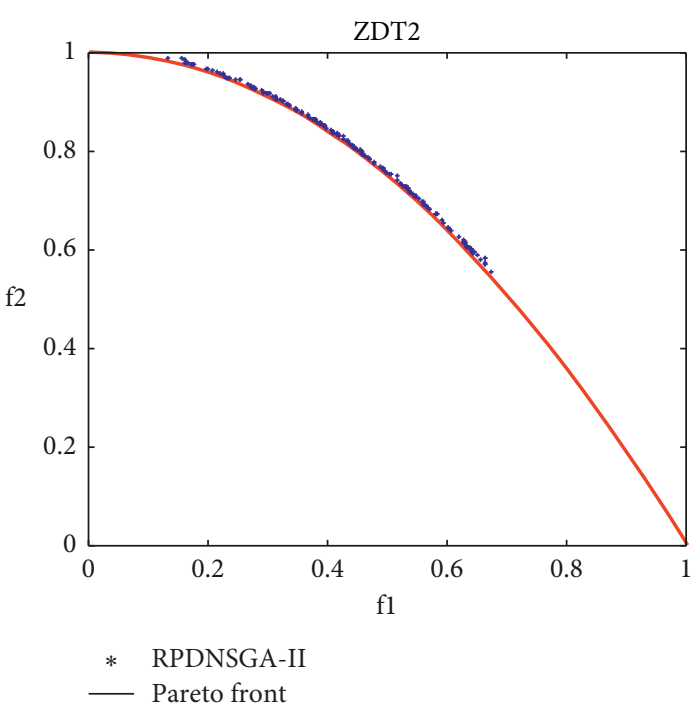

(e)

(f)

FIGURe 3: Obtained results on ZDT2: (a) PF obtained by p-MORSGA on ZDT2; (b) PF obtained by NSGA-II on ZDT2; (c) PF obtained by MOEA/D on ZDT2; (d) PF obtained by MOPSO on ZDT2; (e) PF obtained by rNSGA-II on ZDT2; (f) PF obtained by RPDNSGA-II on ZDT2. 


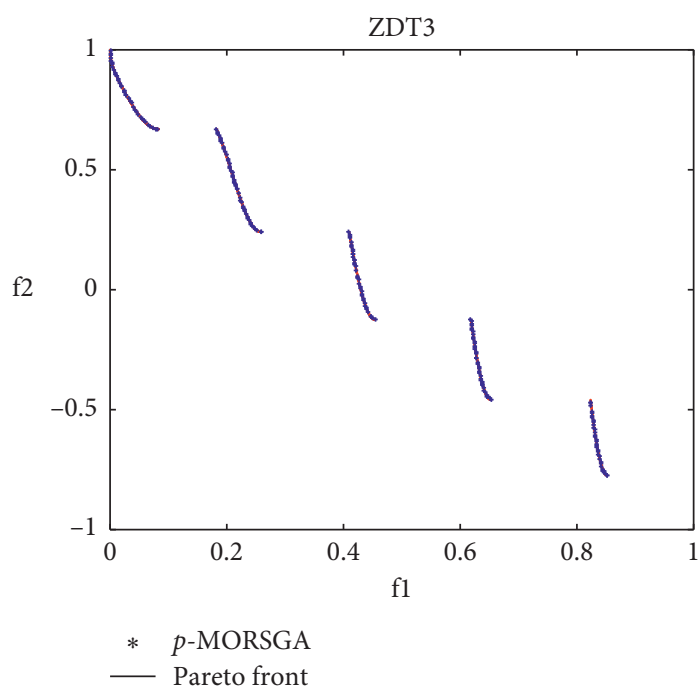

(a)

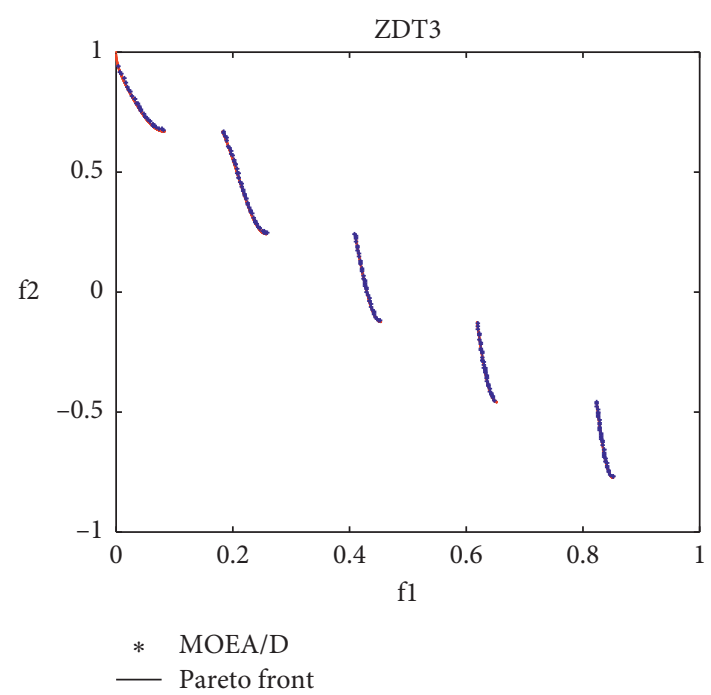

(c)

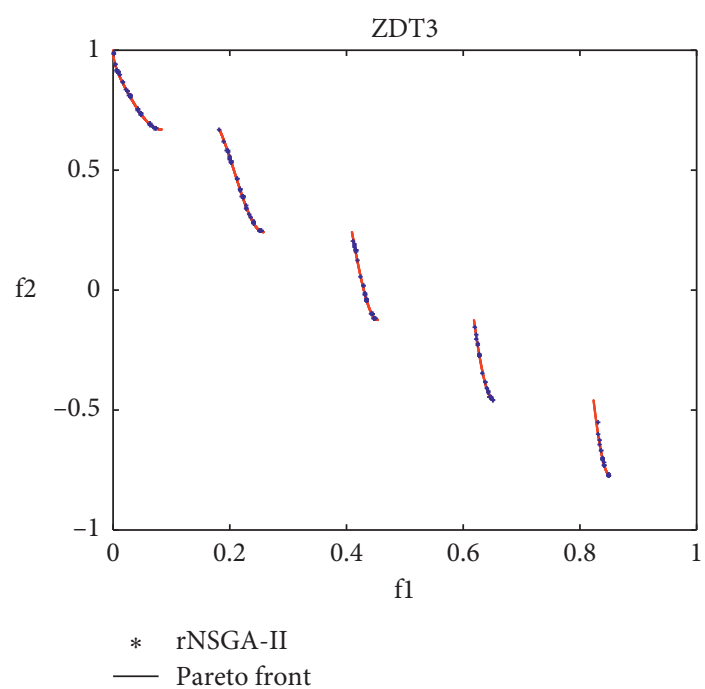

(e)

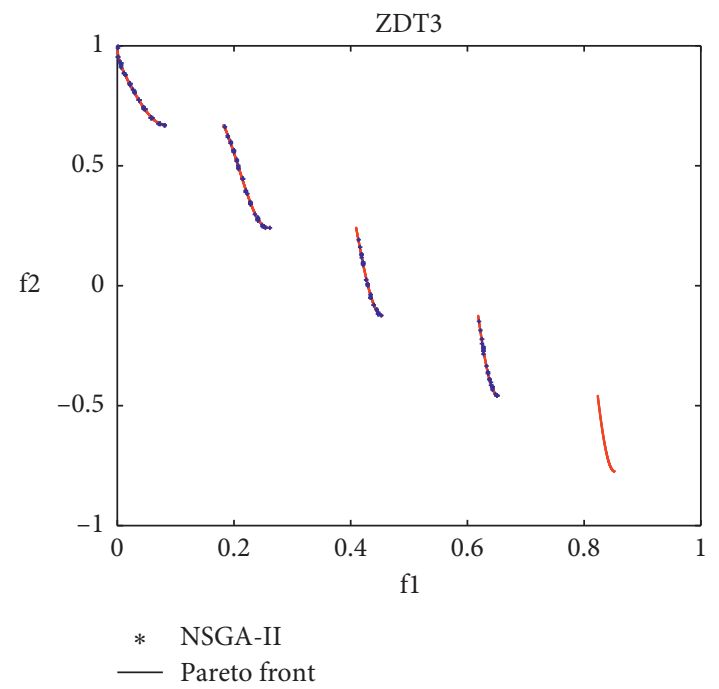

(b)

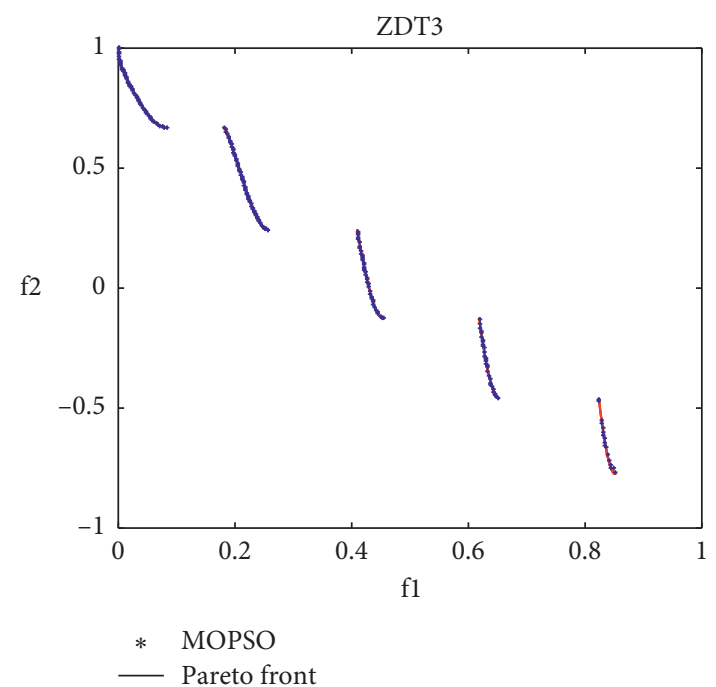

(d)

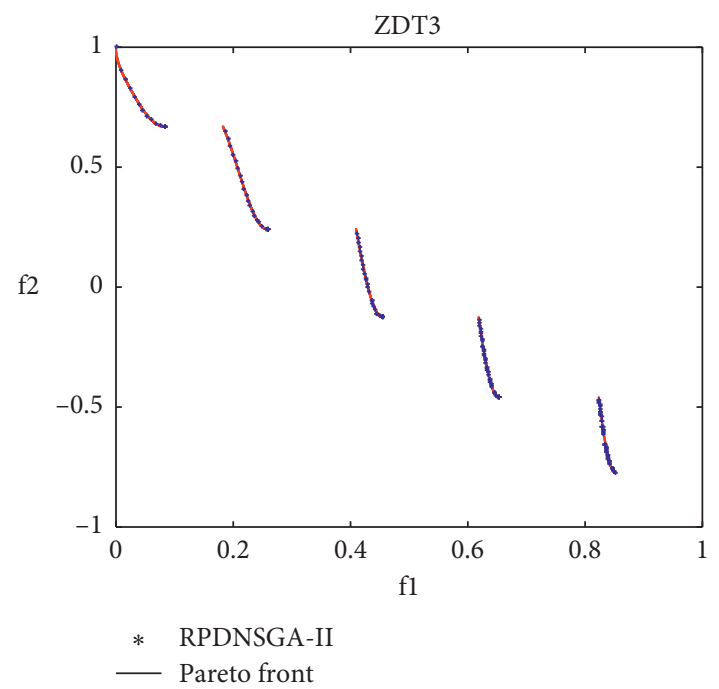

(f)

FIGURe 4: Obtained results on ZDT3: (a) PF obtained by $p$-MORSGA on ZDT3; (b) PF obtained by NSGA-II on ZDT3; (c) PF obtained by MOEA/D on ZDT3; (d) PF obtained by MOPSO on ZDT3; (e) PF obtained by rNSGA-II on ZDT3; (f) PF obtained by RPDNSGA-II on ZDT3. 


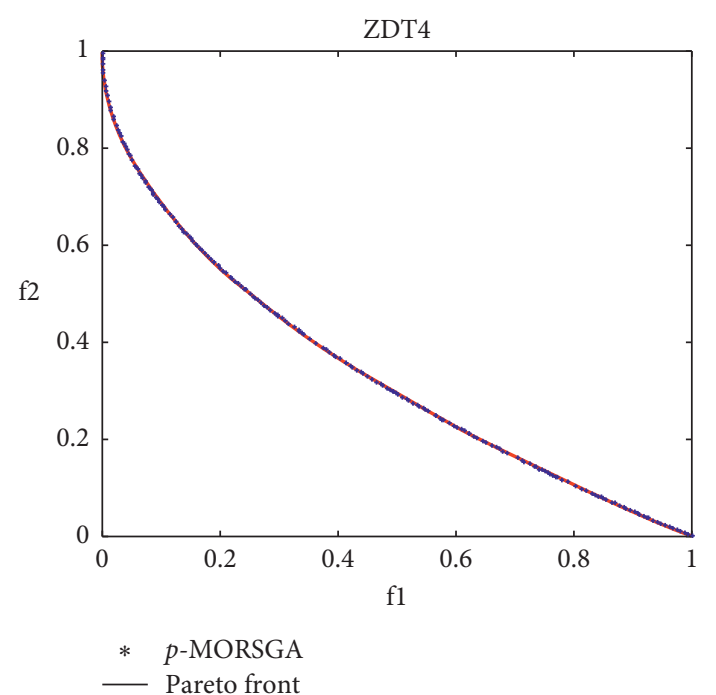

(a)

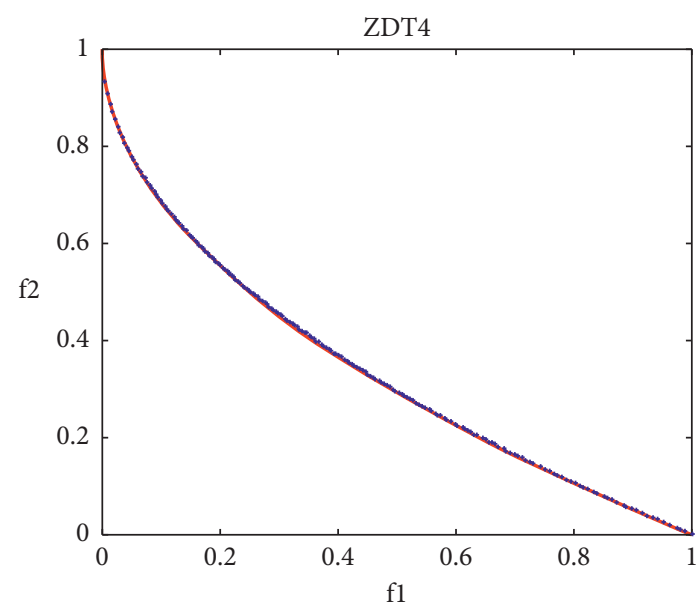

* MOEA/D

— Pareto front

(c)

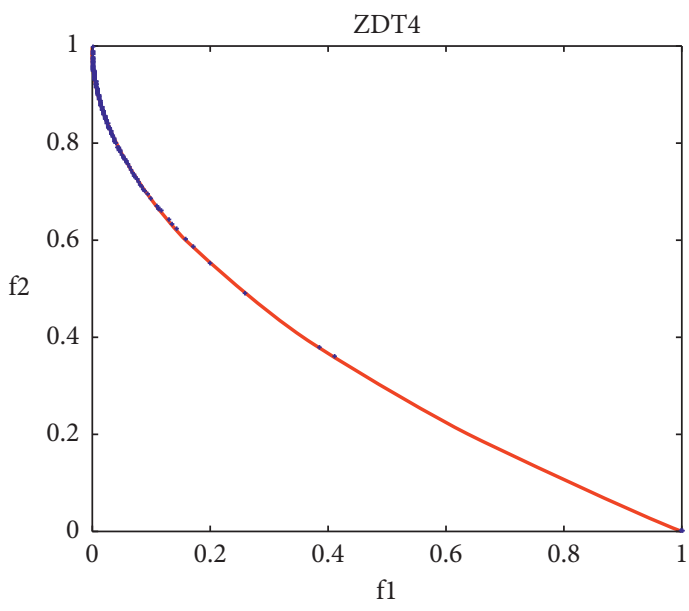

* $\quad$ rNSGA-II

— Pareto front

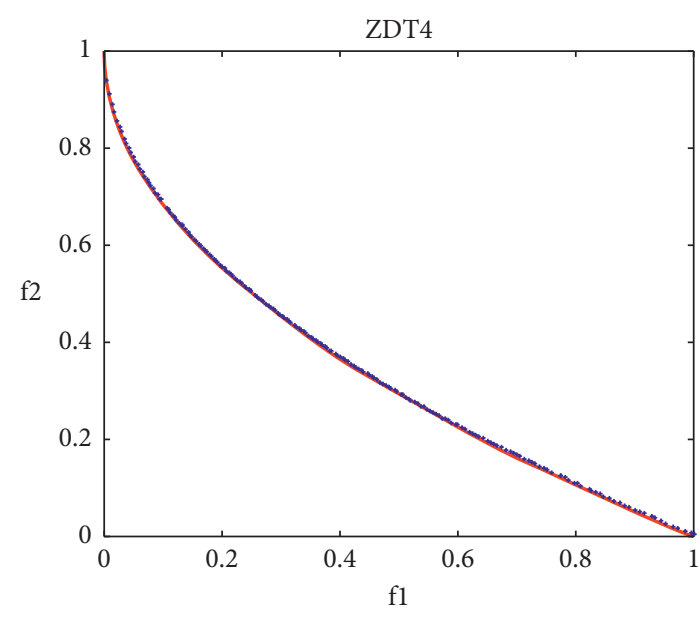

* NSGA-II

_ Pareto front

(b)

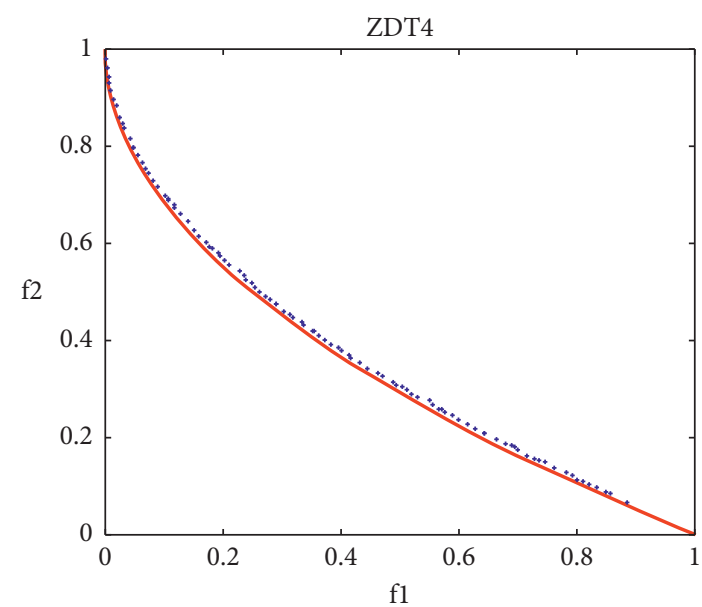

* MOPSO

— Pareto front

(d)

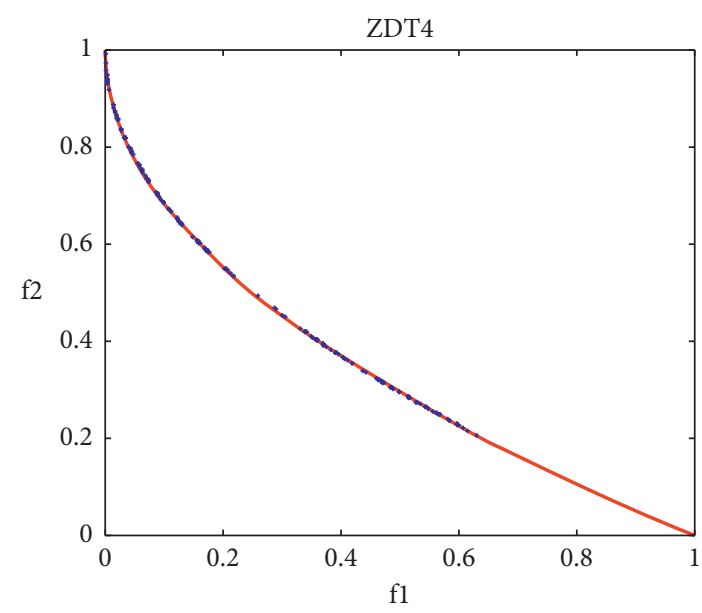

* RPDNSGA-II

- Pareto front

(e)

(f)

FIGURe 5: Obtained results on ZDT4: (a) PF obtained by p-MORSGA on ZDT4; (b) PF obtained by NSGA-II on ZDT4; (c) PF obtained by MOEA/D on ZDT4; (d) PF obtained by MOPSO on ZDT4; (e) PF obtained by rNSGA-II on ZDT4; (f) PF obtained by RPDNSGA-II on ZDT4. 


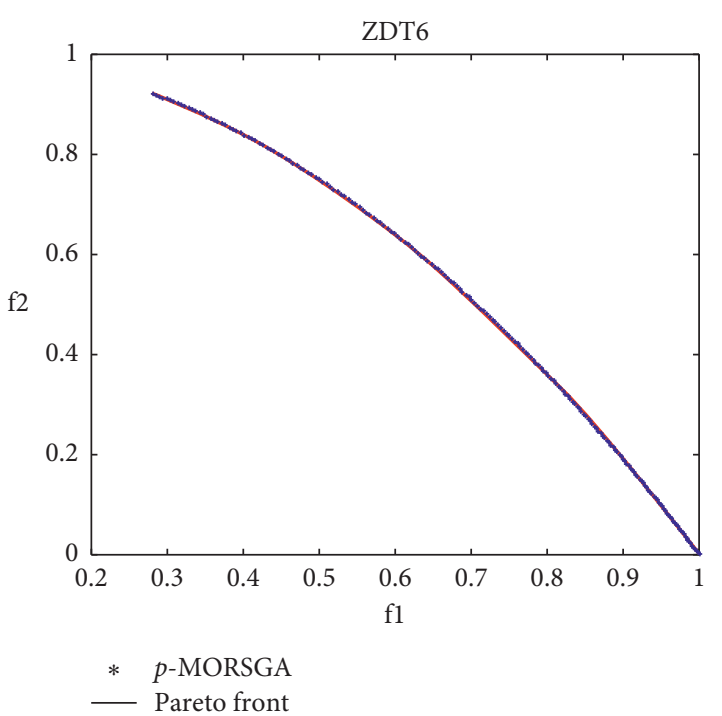

(a)

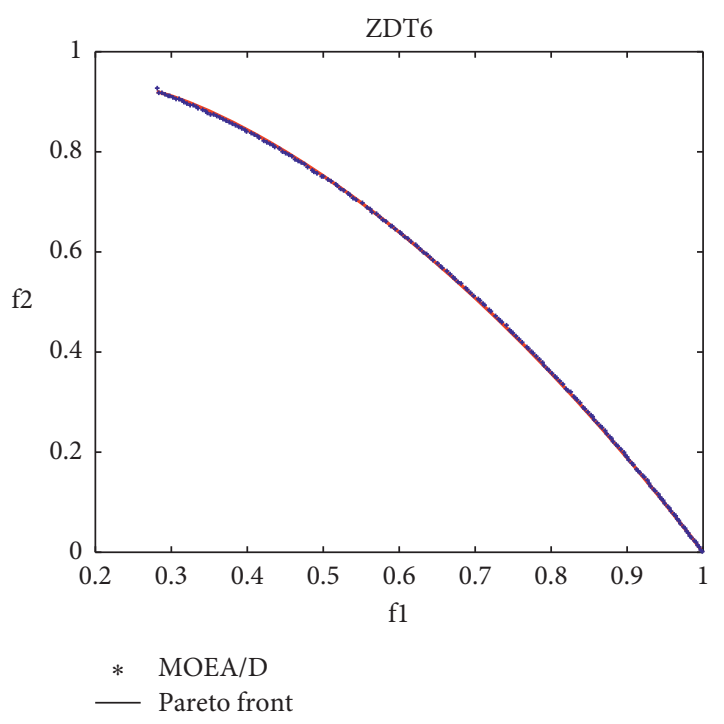

(c)

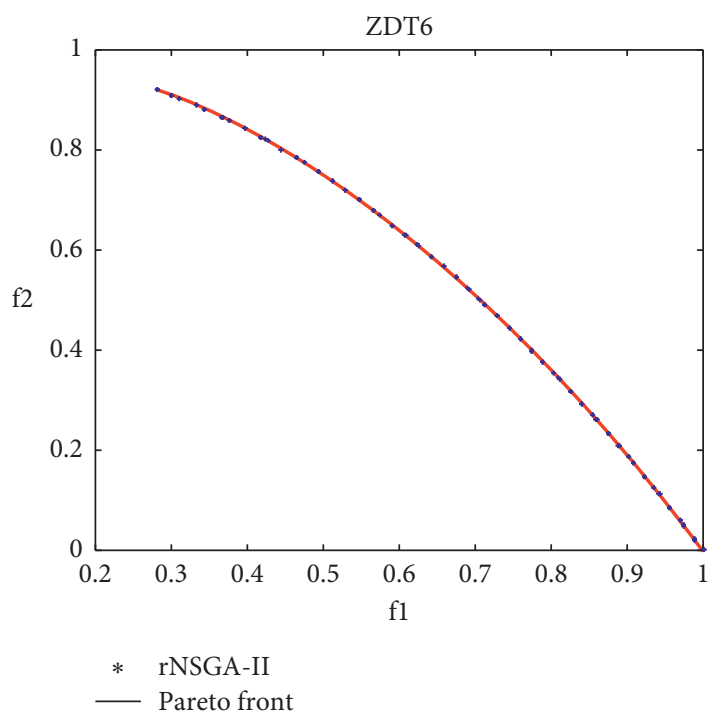

(e)

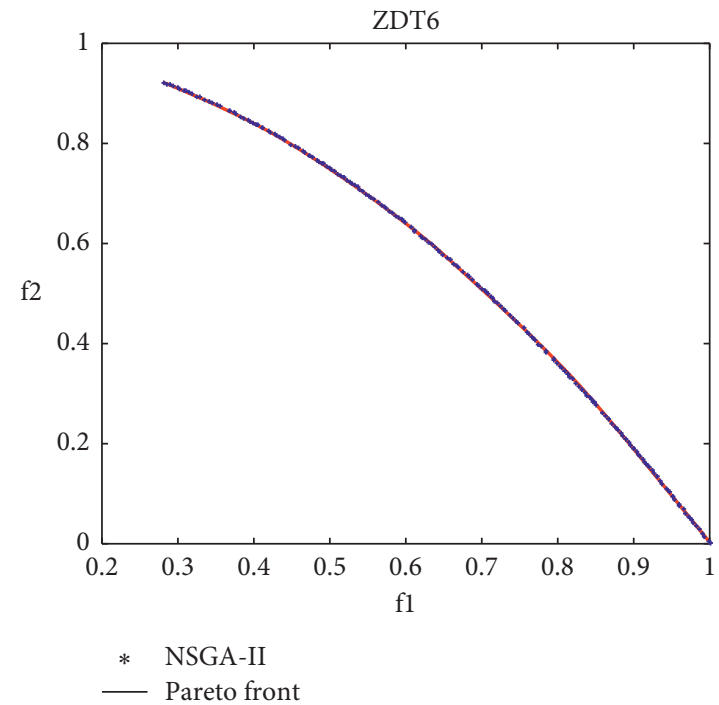

(b)

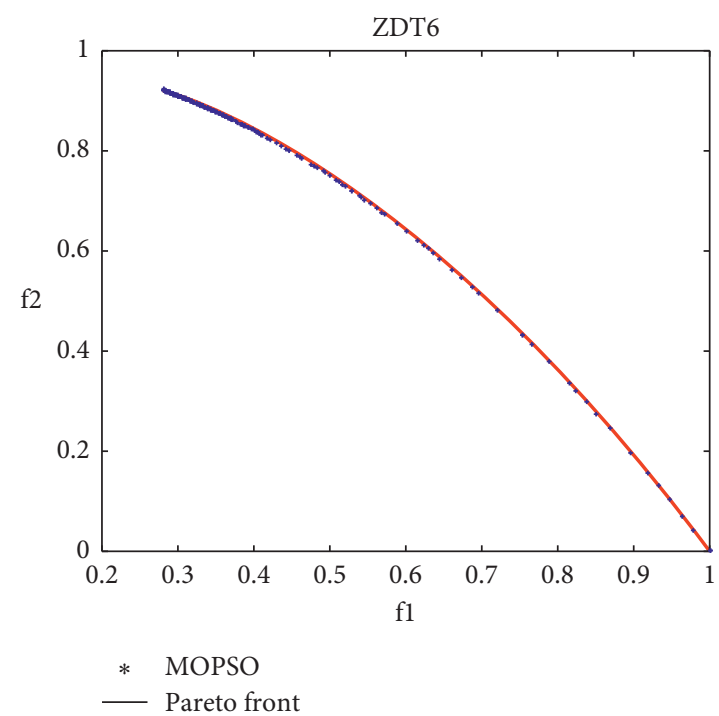

(d)

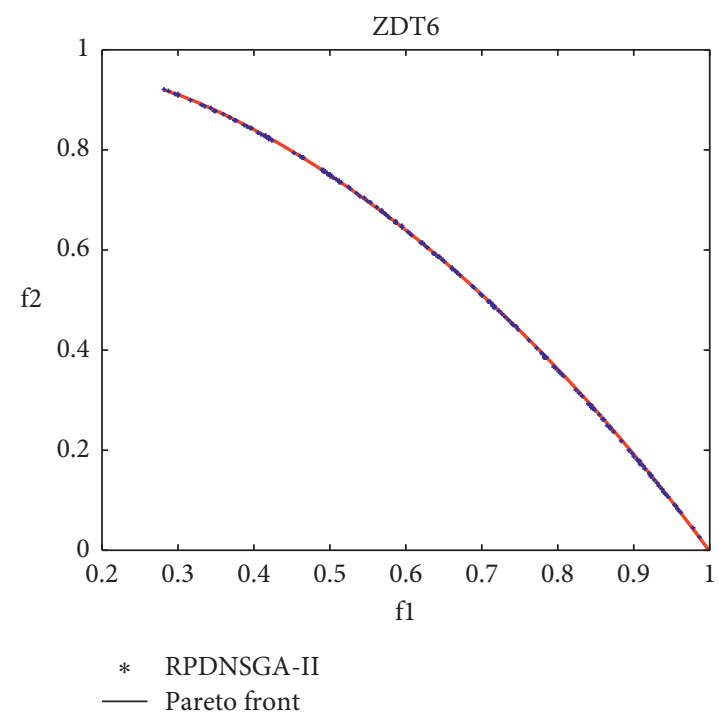

(f)

FIgURE 6: Obtained results on ZDT6: (a) PF obtained by p-MORSGA on ZDT6; (b) PF obtained by NSGA-II on ZDT6; (c) PF obtained by MOEA/D on ZDT6; (d) PF obtained by MOPSO on ZDT6; (e) PF obtained by rNSGA-II on ZDT6; (f) PF obtained by RPDNSGA-II on ZDT6. 
TABLE 2: Test results on three-objective functions.

\begin{tabular}{|c|c|c|c|c|c|c|c|c|}
\hline & Problem & & $p$-MORSGA & NSGA-II & MOEA/D & MOPSO & rNSGA-II & RPDNSGA-II \\
\hline \multirow{8}{*}{ DTLZ1 } & Converge & Avg & $4.9323 e-1$ & $5.2815 e-1$ & $4.6526 \mathrm{e}-1$ & $4.8607 e-1$ & $6.1465 e-1$ & $4.8924 e-1$ \\
\hline & Metric & Std & $4.12 e-1$ & $4.43 e-1$ & $4.00 e-1$ & $1.14 e-1$ & $3.25 e-1$ & $4.06 e-1$ \\
\hline & Diversity & Avg & $1.0484 \mathrm{e}-1$ & $2.6981 e-1$ & $1.3155 e-1$ & $2.9024 e+1$ & $2.0744 e-1$ & $1.5770 e-1$ \\
\hline & Metric & Std & $1.23 \mathrm{e}-1$ & $2.94 e-1$ & $2.32 e-1$ & $6.94 e+0$ & $2.51 e-1$ & $1.74 e-1$ \\
\hline & IGD & Avg & $1.0484 \mathrm{e}-1$ & $1.9798 e-1$ & $1.1472 e-1$ & $7.6192 e+0$ & $1.7254 e-1$ & $1.3049 e-1$ \\
\hline & Metric & Std & $1.23 e-1$ & $2.09 e-1$ & $1.91 e-1$ & $4.36 e+0$ & $2.03 e-1$ & $1.42 e-1$ \\
\hline & $\mathrm{HV}$ & Avg & $6.5272 \mathrm{e}-1$ & $4.6727 e-1$ & $6.5240 e-1$ & $1.0000 e+0$ & $5.2542 e-1$ & $5.6318 e-1$ \\
\hline & Metric & Std & $3.52 \mathrm{e}-1$ & $3.92 e-1$ & $3.04 e-1$ & $0.00 e+0$ & $3.70 e-1$ & $3.48 e-1$ \\
\hline \multirow{8}{*}{ DTLZ2 } & Converge & Avg & $7.9873 e-1$ & $7.1867 e-1$ & $6.1309 e-3$ & $8.5567 e-1$ & $3.1040 e-2$ & $1.6016 e-1$ \\
\hline & Metric & Std & $6.32 e-2$ & $8.71 e-2$ & $9.73 e-3$ & $5.94 e-2$ & $2.43 e-2$ & $3.91 e-2$ \\
\hline & Diversity & Avg & $4.3032 \mathrm{e}-2$ & $5.8855 e-2$ & $5.9884 e-2$ & $8.3690 e-2$ & $5.0484 e-1$ & $4.6103 e-2$ \\
\hline & Metric & Std & $1.10 \mathrm{e}-2$ & $1.65 e-2$ & $1.88 e-2$ & $3.28 e-2$ & $1.14 e-2$ & $1.37 e-2$ \\
\hline & IGD & Avg & $4.3032 \mathrm{e}-2$ & $5.8855 e-2$ & $5.9884 e-2$ & $8.3690 e-2$ & $5.0484 e-1$ & $4.6103 e-2$ \\
\hline & Metric & Std & $1.10 \mathrm{e}-2$ & $1.65 e-2$ & $1.88 e-2$ & $3.28 e-2$ & $1.14 e-2$ & $1.37 e-2$ \\
\hline & $\mathrm{HV}$ & Avg & $5.5239 \mathrm{e}-1$ & $5.4209 e-1$ & $5.4279 e-1$ & $4.8186 e-1$ & $1.3043 e-1$ & $5.6408 e-1$ \\
\hline & Metric & Std & $1.69 \mathrm{e}-2$ & $1.78 e-2$ & $1.74 e-2$ & $5.18 e-2$ & $7.24 e-3$ & $1.19 e-2$ \\
\hline \multirow{8}{*}{ DTLZ3 } & Converge & Avg & $2.6323 e-1$ & $5.2444 e-1$ & $4.8054 e-1$ & $2.7995 e-1$ & $6.3365 e-1$ & $5.7198 e-1$ \\
\hline & Metric & Std & $1.00 \mathrm{e}-1$ & $2.51 e-1$ & $1.62 e-1$ & $1.08 e-1$ & $2.61 e-1$ & $1.34 e-1$ \\
\hline & Diversity & Avg & $5.0122 \mathrm{e}+0$ & $5.1430 e+0$ & $6.8767 e+0$ & $2.4610 e+2$ & $5.3446 e+0$ & $8.3516 e+0$ \\
\hline & Metric & Std & $4.32 \mathrm{e}+0$ & $5.96 e+0$ & $7.45 e+0$ & $5.51 e+1$ & $5.62 e+0$ & $8.37 e+0$ \\
\hline & IGD & Avg & $4.4200 \mathrm{e}+0$ & $4.4432 e+0$ & $6.1109 e+0$ & $1.4399 e+2$ & $4.7071 e+0$ & $4.4227 e+0$ \\
\hline & Metric & Std & $4.11 \mathrm{e}+0$ & $5.08 e+0$ & $6.91 e+0$ & $6.48 e+1$ & $4.91 e+0$ & $4.28 e+0$ \\
\hline & $\mathrm{HV}$ & Avg & $2.3310 \mathrm{e}-1$ & $2.2438 e-1$ & $2.2523 e-1$ & $1.0000 e+0$ & $2.2284 e-1$ & $2.2596 e-1$ \\
\hline & Metric & Std & $2.343-1$ & $2.90 e-1$ & $2.91 e-1$ & $0.00 e+0$ & $2.88 e-1$ & $2.92 e-1$ \\
\hline
\end{tabular}

of $p$-MORSGA and other three MOEAs are shown in Figures 8-10. Table 2 shows the test result on three-objective experience.

It can be seen from Figure 8 that $p$-MORSGA and NSGA-II have relatively complete edge shapes, the MOEA/D algorithm's distribution is relatively uniform, followed by rNSGA-II, and MOPSO and RPDNSGA-II do not get a good frontier.

From Figure 9, p-MORSGA and MOEA/D have obtained a good frontier, and the MOEA/D distribution is uniform. As can be seen from Table 2, p-MORSGA has better convergence and diversity. As can be seen from Figure 10, p-MORSGA can get a better solution, followed by MOEA/D. In addition, MOEA's performance is slightly worse than that of $p$-MORSGA. However, $p$-MORSGA is superior to MOEAs for the problem.

4.3. Time Complexity Analysis. In order to demonstrate the difference in the time complexity of these algorithms, Figures 11 and 12 plot the average CPU time over 10 runs. Figure 11 shows the CPU time for six algorithms and gives the results on five benchmark functions (ZDT1, ZDT2, ZDT3, ZDT4, and ZDT6). Overall, rNSGA-II performs poorly, and the running time is 3.02-3.56 times that of other algorithms. The time complexity of $p$-MORSGA is better than rNSGA-II and RPDNSGA-II and worse than NSGA-II. NSGA-II has the best performance with time complexity, and $p$-MORSGA is about 1.04-1.49 times that of NSGA-II. It is worth mentioning that although $p$-MORSGA consumes more running time than NSGA-II, the performance has been significantly improved.

Figure 12 shows the CPU time for six algorithms and gives the results on three benchmark functions (DTLZ1, DTLZ2, and DTLZ3). There is some difference as the number of objectives increases. The time complexity of rNSGA-II and other algorithms is reduced. rNSGA-II is about 1.01 to 2.67 times that of other algorithms. In each test function, the best performance of time complexity is still NSGA-II. Secondly, the best performance of time complexity is $p$-MORSGA, and $p$-MORSGA is about 1.02-1.26 times that of NSGA-II.

Compared to Figure 11, the running time of the algorithm increases overall. It can be seen that the threeobjective benchmark functions are more difficult to calculate than the two-objective benchmark functions.

\section{5. $p$-MORSGA for Multiobjective Applications}

\subsection{Introduction of $M O P O P$}

5.1.1. Two-Objective Portfolio Model. Markowitz [38] proposes an MV portfolio model, which is a formal twoobjective portfolio model. The model uses the covariance and average return of assets to describe the risk and return of investment, respectively. Two conflicting aspects are considered by this model: maximizing the portfolio's expected return while minimizing its risk [39]. The MV model is constructed as follows [38]: 


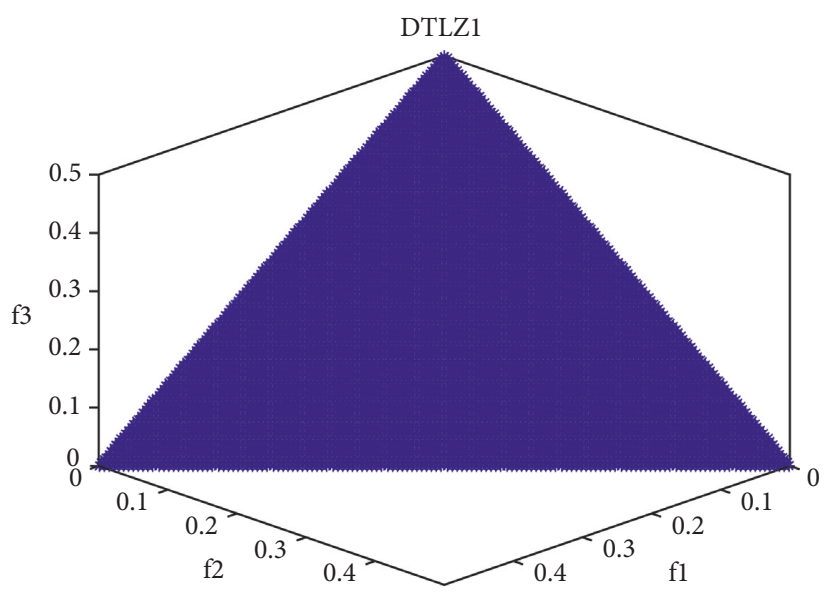

(a)

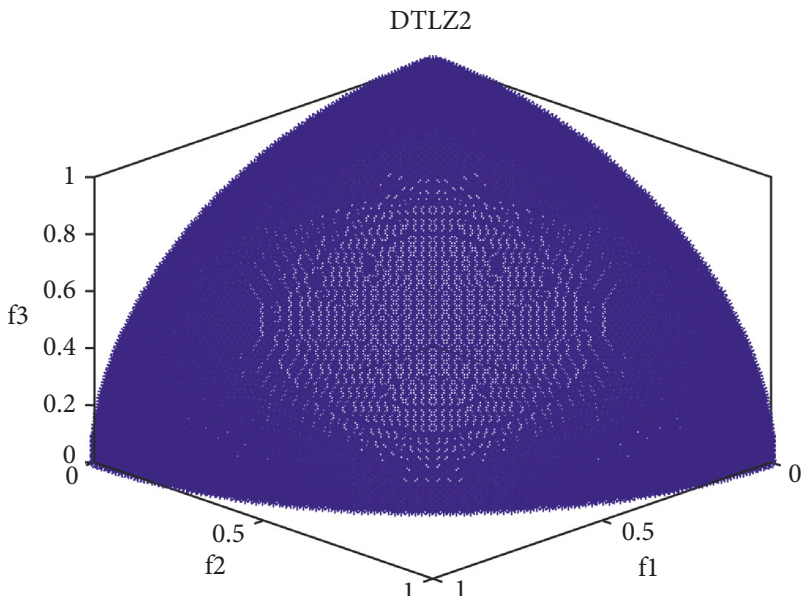

(b)

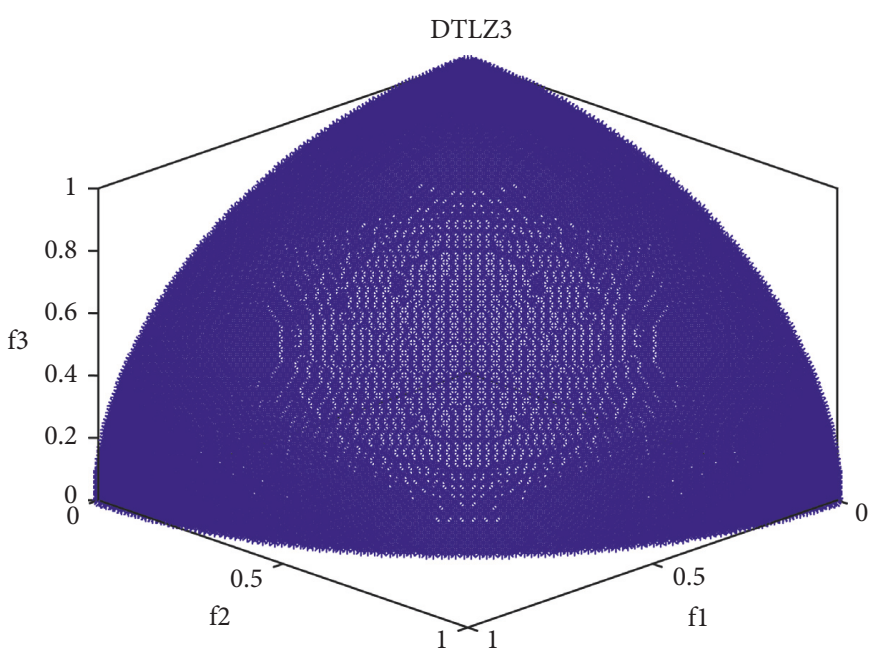

(c)

Figure 7: True PF on DTLZ functions. (a) True PF on DTLZ1. (b) True PF on DTLZ2. (c) True PF on DTLZ3.

$$
\begin{array}{ll}
\text { Min } & \sigma^{2}=\sum_{i=1}^{N} \sum_{j=1}^{N} w_{i} w_{j} \sigma_{i j}, \quad \text { minimizing risk, } \\
\text { Max } & r=\sum_{i=1}^{N} w_{i} r_{i}, \quad \text { maximizing expected return, } \\
\text { subject to } & \sum_{i=1}^{N} w_{i}=1, \quad 0 \leq w_{i} \leq 1, i=1,2, \ldots, N, \\
& \text { budget constraint, }
\end{array}
$$

where $\sigma^{2}$ are portfolios, $w_{i}$ and $w_{j}$ are the weights, $\sigma_{i j}$ expresses covariance between two assets, $N$ denotes quantity, and $r_{i}$ indicates expected return of the $i$ th asset.

The assumptions of several portfolio models introduced are too harsh to meet the actual needs of the securities market, which leads to the deviation between the results of the model operation and the actual situation. On the basis of revising the hypothesis of the classical portfolio model, semivariance is introduced to replace variance, which makes the model more reasonable, more in line with the investment and financing environment of China's financial market, and provides more effective auxiliary tools for investors.

5.1.2. Three-Objective Portfolio Model. Different from the 2objective model, the 3-objective portfolio model includes other objective: minimizing expected cost.

The MV model has the function as follows:

$$
\begin{aligned}
& \sum_{i=1}^{N} w_{i}=1, \quad a_{i} z_{i} \leq w_{i} \leq b_{i} z_{i}, i=1,2, \ldots, N, \\
& 0 \leq a_{i} \leq 1,0 \leq b_{i} \leq 1, \\
& z_{i}= \begin{cases}1, & \text { for } w_{i}>0 \\
0, & \text { otherwise }\end{cases} \\
& \sum_{i=1}^{N} z_{i}=K
\end{aligned}
$$

The weight of each asset portfolio should be the sum of 1 . 


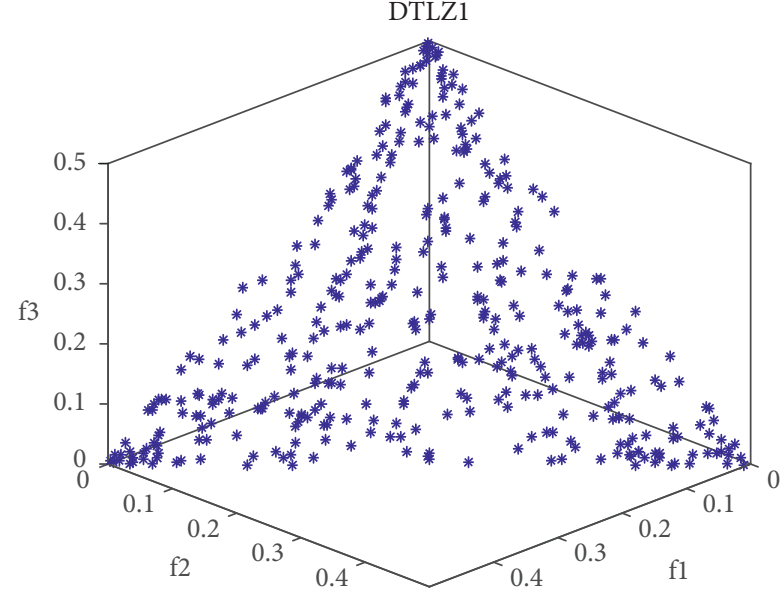

(a)

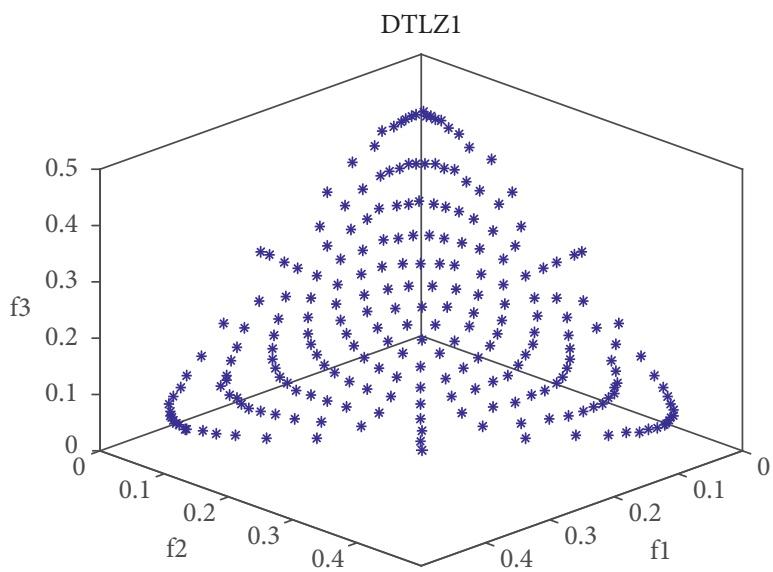

(c)

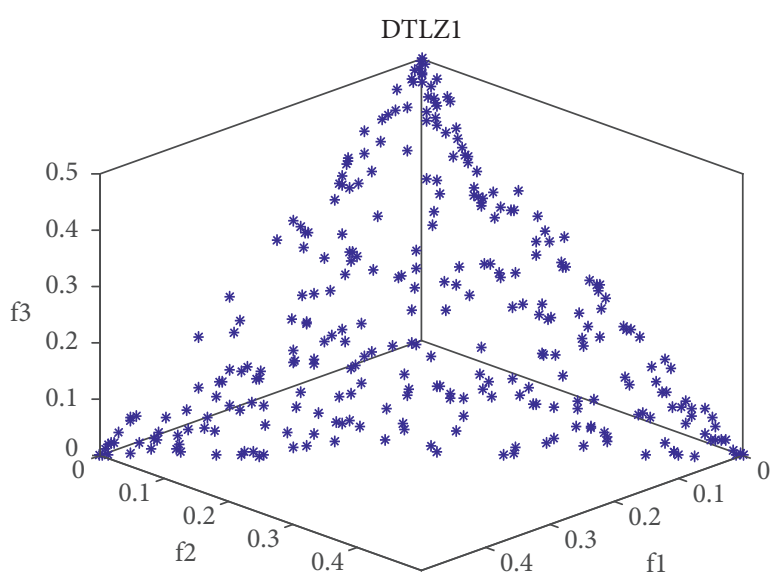

(e)

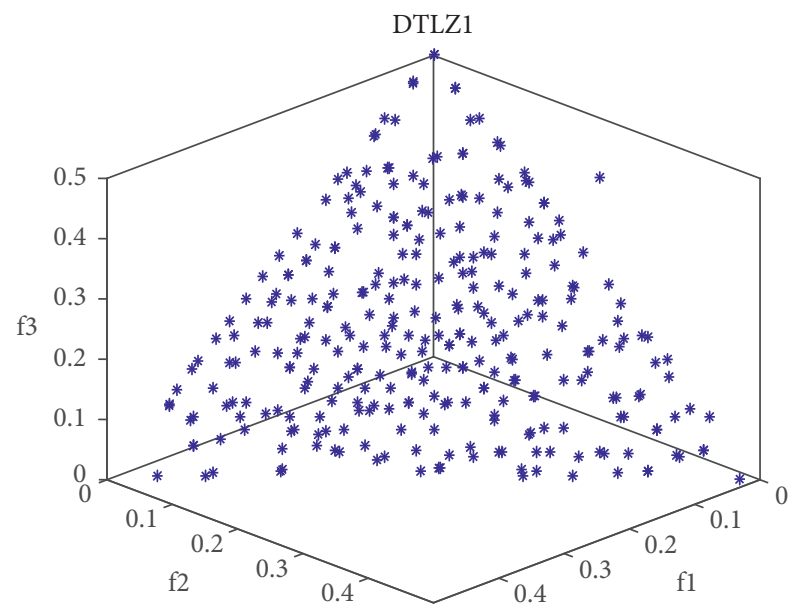

(b)

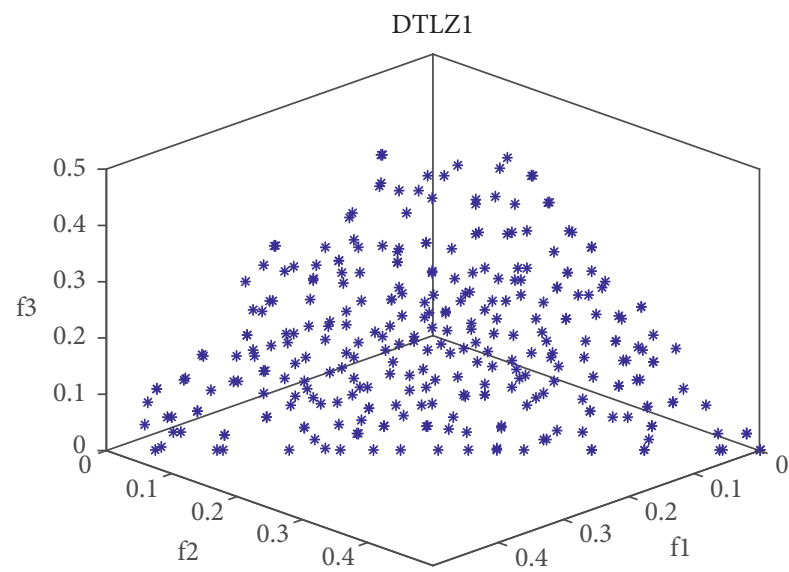

(d)

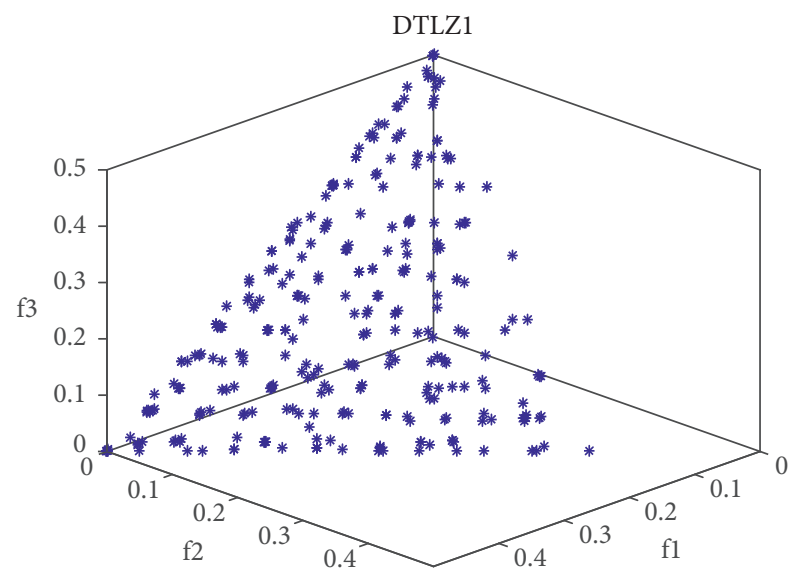

(f)

FIGUre 8: Obtained results on DTLZ1: (a) PF obtained by p-MORSGA on DTLZ1; (b) PF obtained by NSGA-II on DTLZ1; (c) PF obtained by MOEA/D on DTLZ1; (d) PF obtained by MOPSO on DTLZ1; (e) PF obtained by rNSGA-II on DTLZ1; (f) PF obtained by RPDNSGA-II on DTLZ1. 


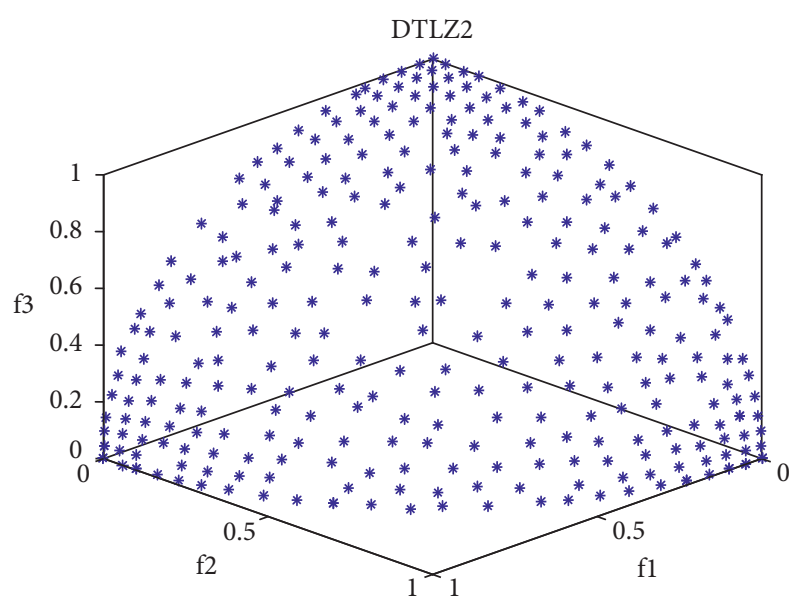

(a)

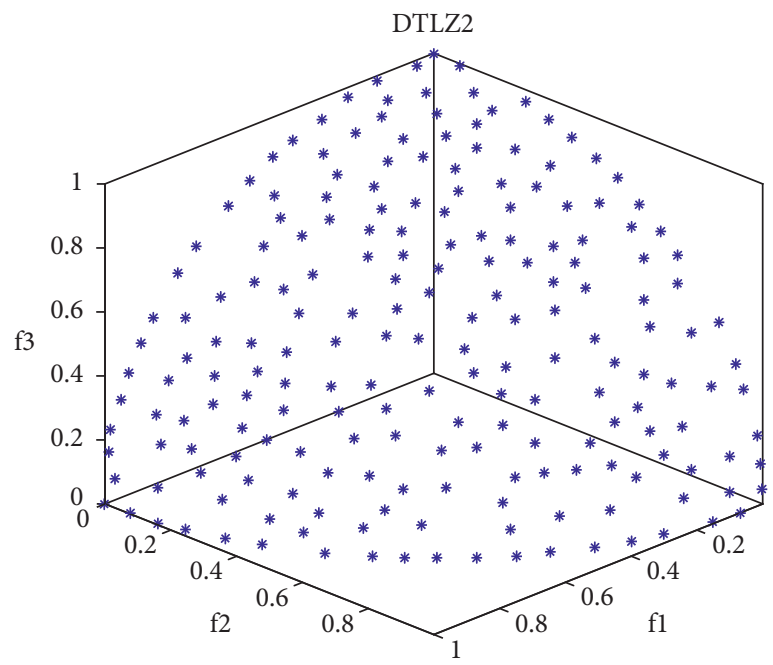

(c)

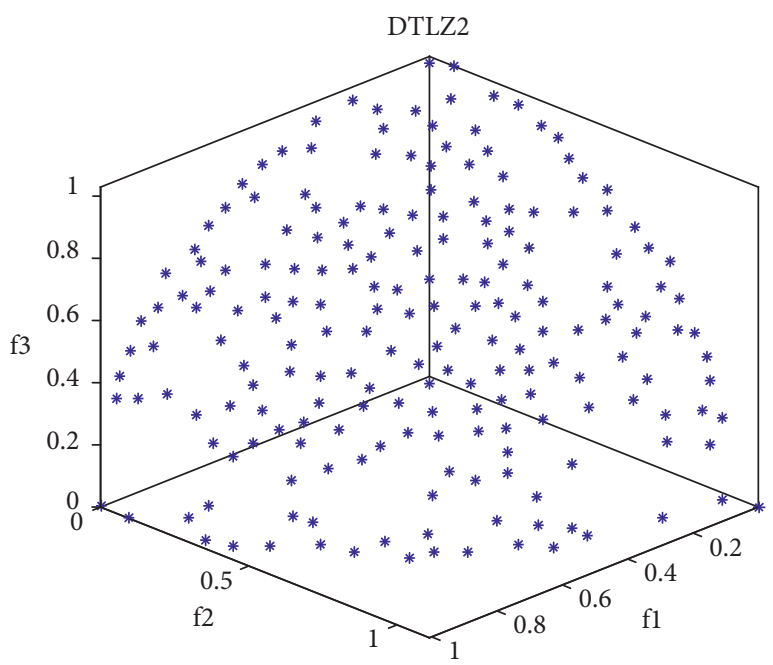

(e)

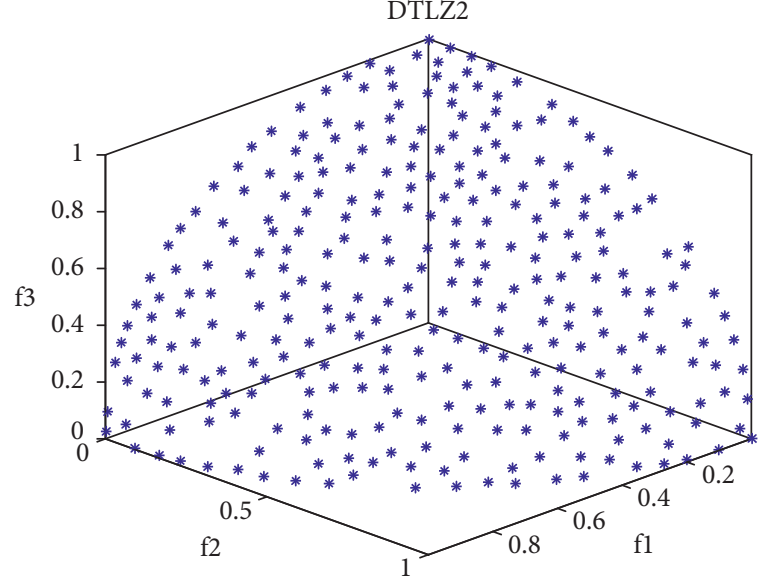

(b)

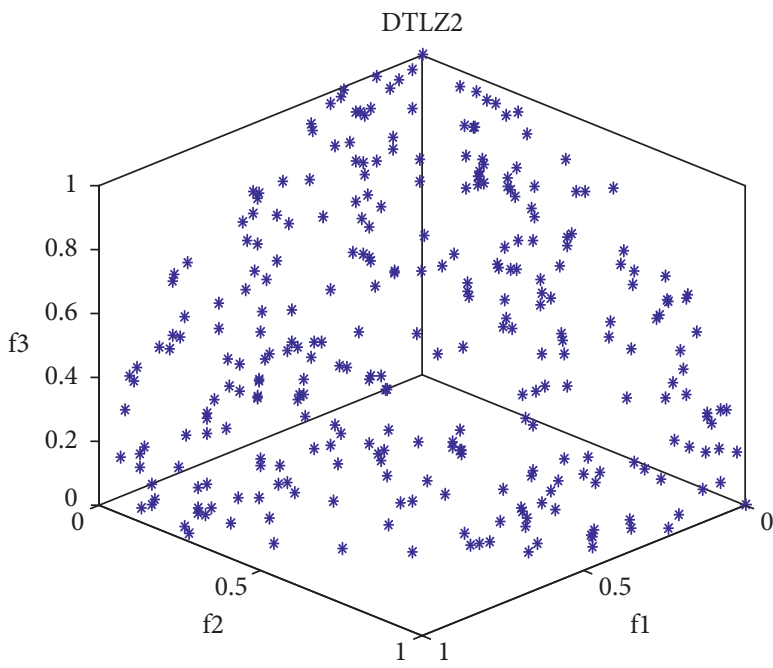

(d)

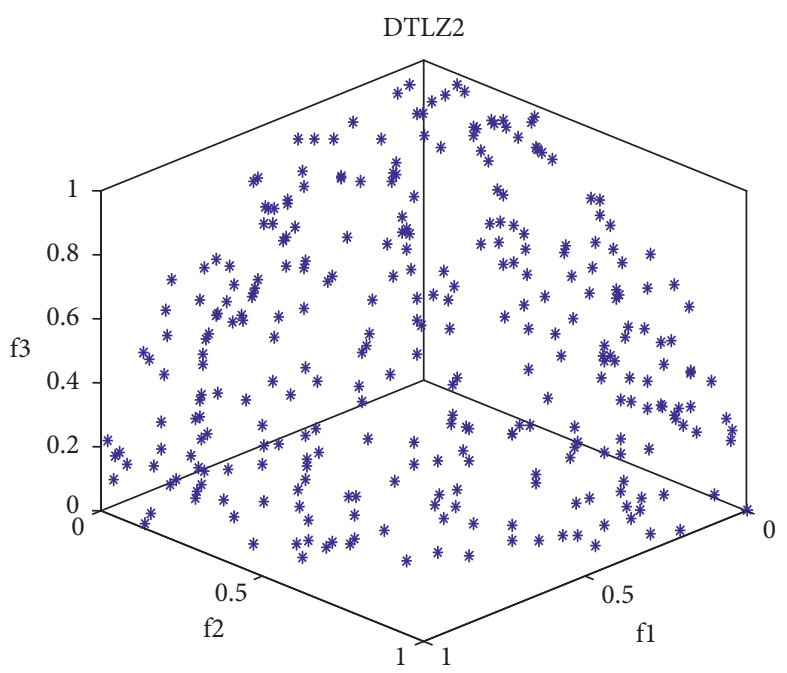

(f)

FIgure 9: Obtained results on DTLZ2: (a) PF obtained by p-MORSGA on DTLZ2; (b) PF obtained by NSGA-II on DTLZ2; (c) PF obtained by MOEA/D on DTLZ2; (d) PF obtained by MOPSO on DTLZ3; (e) PF obtained by rNSGA-II on DTLZ2; (f) PF obtained by RPDNSGA-II on DTLZ2. 


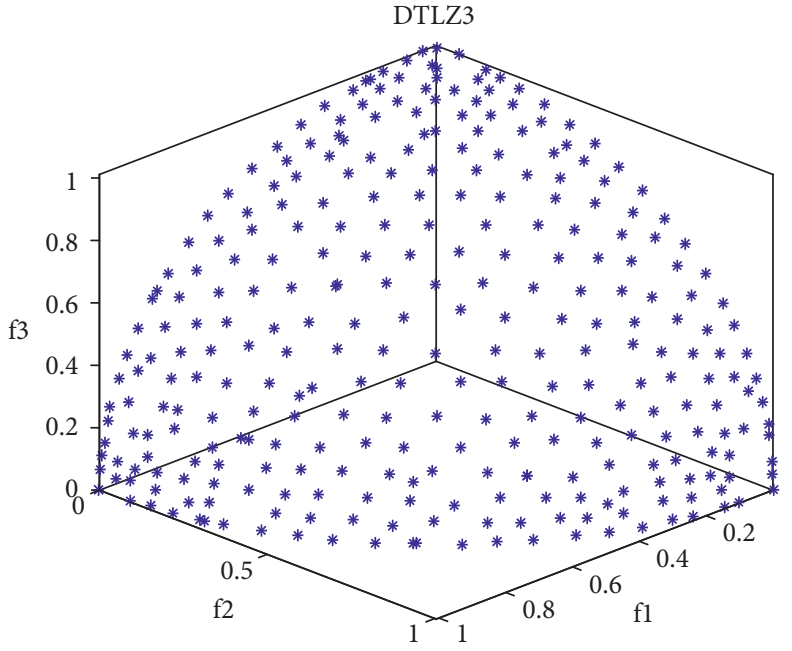

(a)

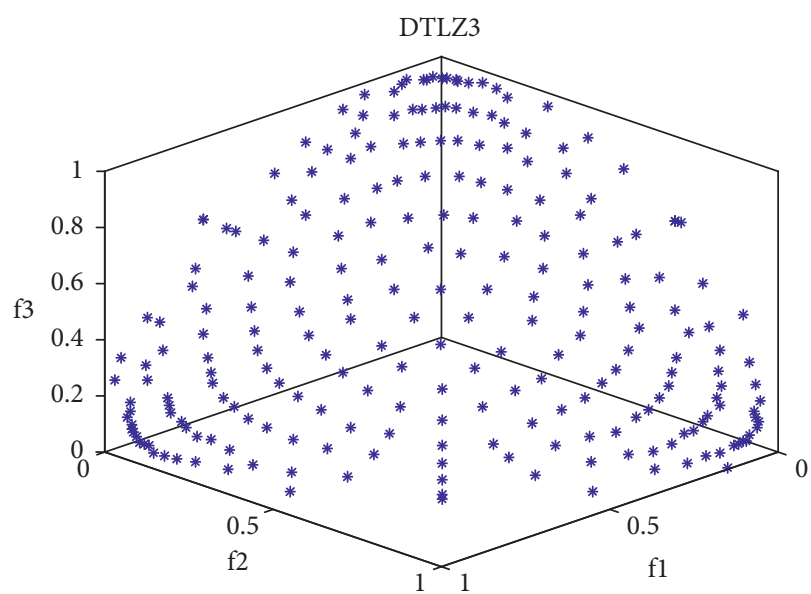

(c)

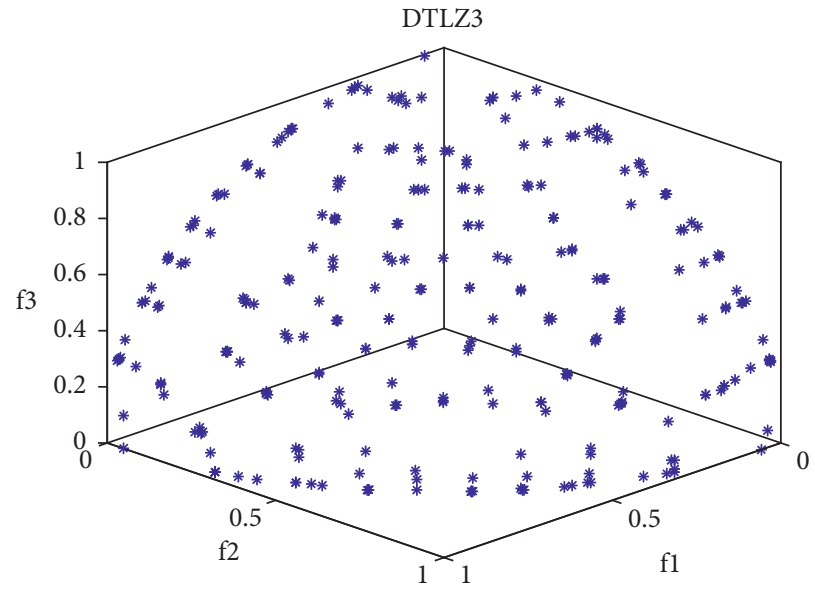

(e)

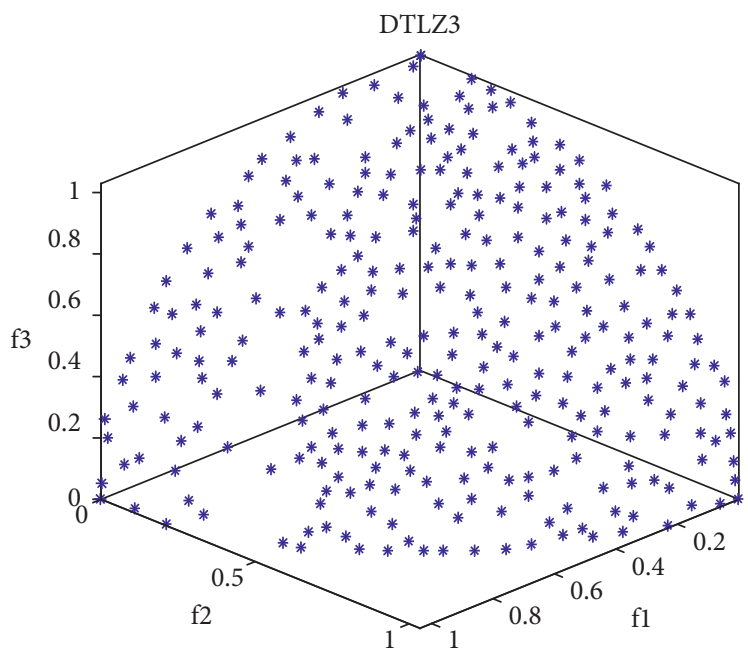

(b)

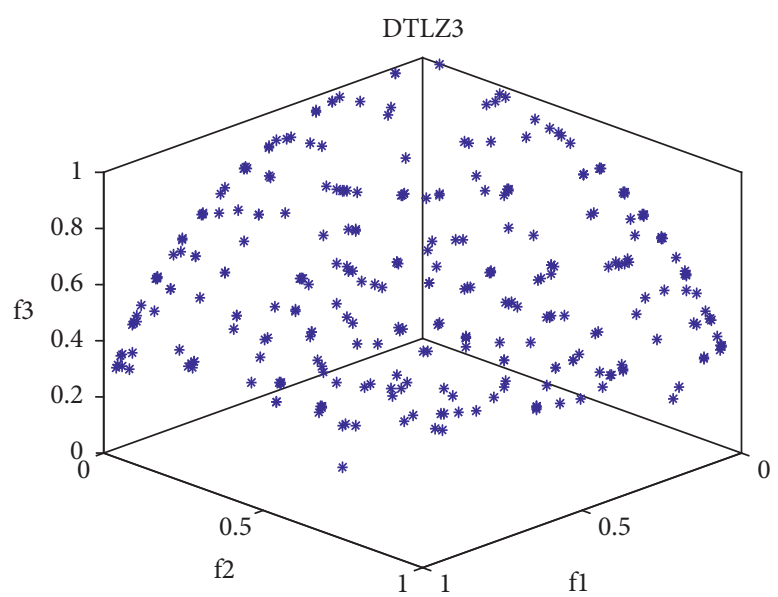

(d)

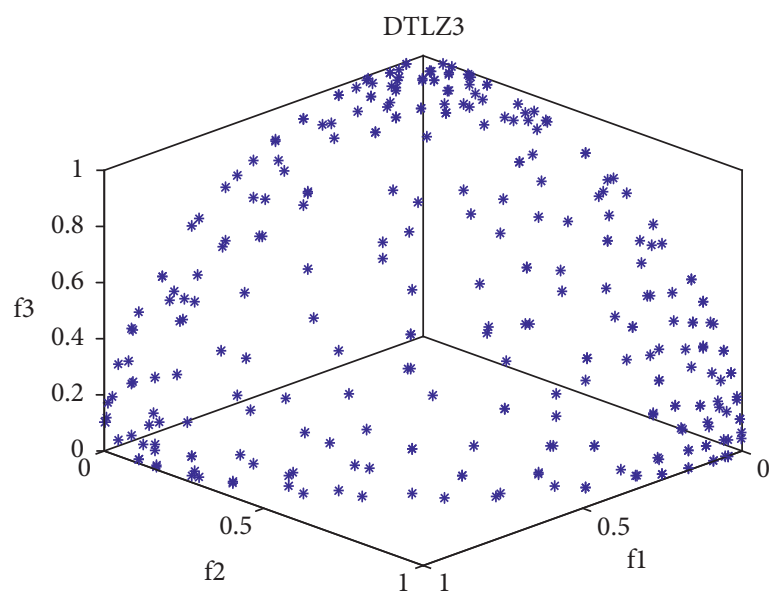

(f)

FIGURe 10: Obtained results on DTLZ3: (a) PF obtained by $p$-MORSGA on DTLZ3; (b) PF obtained by NSGA-II on DTLZ3; (c) PF obtained by MOEA/D on DTLZ3; (d) PF obtained by MOPSO on DTLZ3; (e) PF obtained by rNSGA-II on DTLZ3; (f) PF obtained by RPDNSGA-II on DTLZ2. 


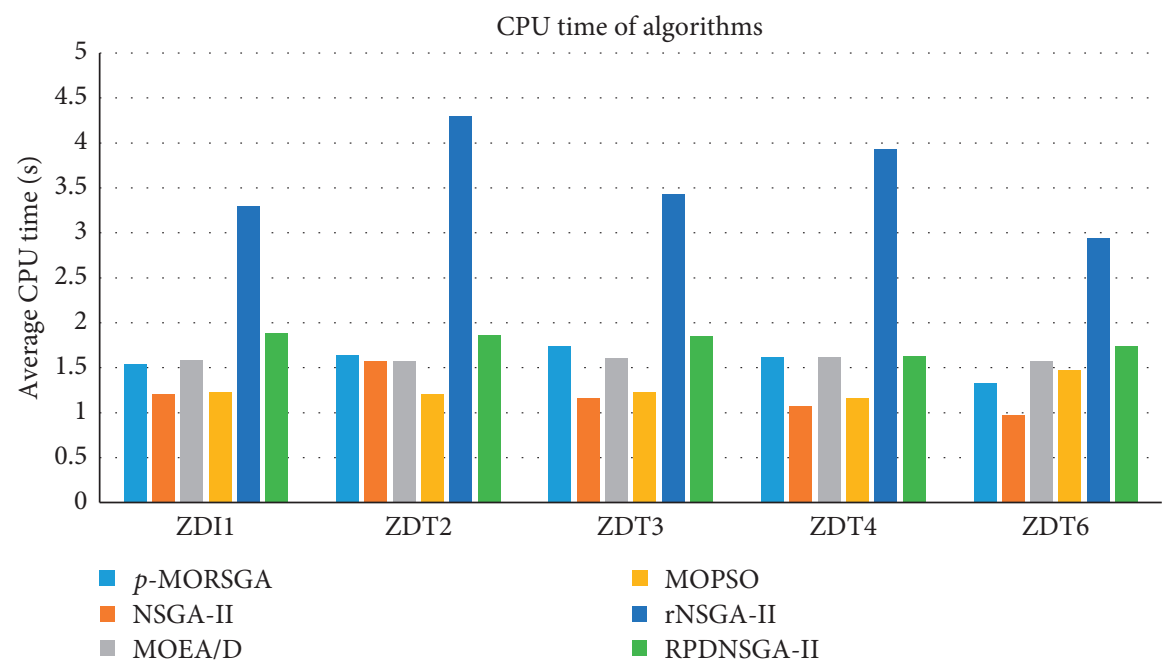

Figure 11: CPU time of MOEAs on two-objective benchmark functions.

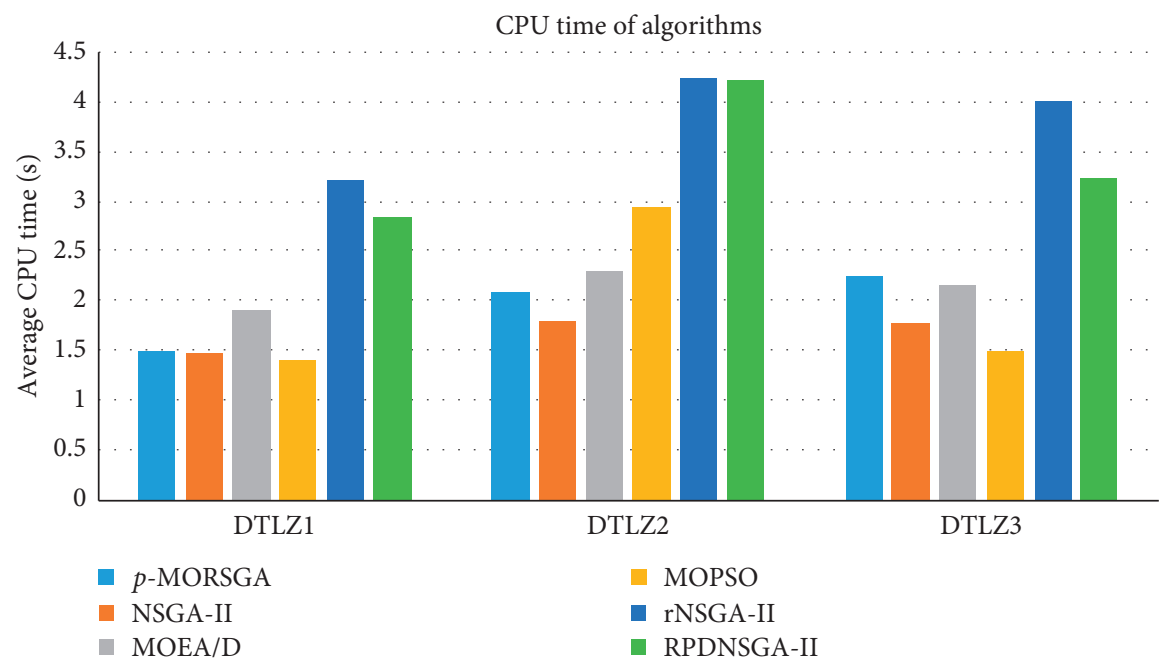

Figure 12: CPU time of MOEAs on three-objective benchmark functions.

In [39], semivariance is described as follows:

$$
\begin{aligned}
\sum_{i j B} & =E\left\{\operatorname{Min}\left(R_{i}-B, 0\right) \cdot \operatorname{Min}\left(R_{j}-B, 0\right)\right\} \\
& =\left(\frac{1}{T}\right) \cdot \sum_{i=1}^{T}\left[\operatorname{Min}\left(R_{i t}-B, 0\right) \cdot \operatorname{Min}\left(R_{j t}-B, 0\right)\right],
\end{aligned}
$$

where $B$ represents the comparative return and $R_{i t}$ represents the earnings of asset $I$ at period $T$.

The indirect method is utilized to represent transaction cost:

$$
d\left(W_{i}, W_{j}\right)=\sqrt{\sum_{n=1}^{N}\left(w_{n}^{i}-w_{n}^{j}\right)^{2}} .
$$

Therefore, the return-risk-cost portfolio model is constructed as follows:
$\operatorname{Max} E=\sum_{i=1}^{N} w_{i} r_{i}, \quad$ maximizing expected return,

Min $R=\sum_{i=1}^{N} \sum_{j=1}^{N} w_{i} w_{j} \sum i j, \quad$ minimizing risk,

Min $\quad C=\sqrt{\sum_{n=1}^{N}\left(w_{n}^{i}-w_{n}^{j}\right)^{2}}, \quad$ minimizing expected cost.

Budget constraints are given as follows: 


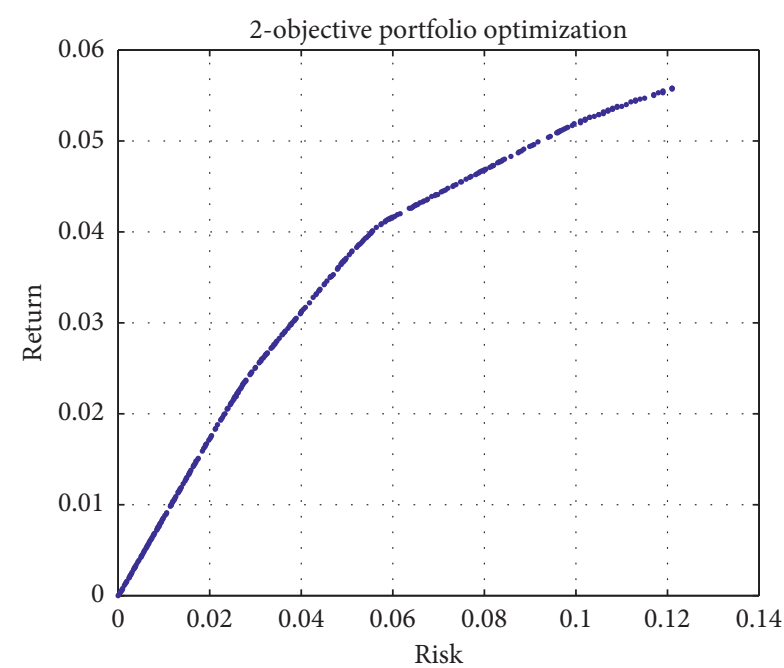

(a)

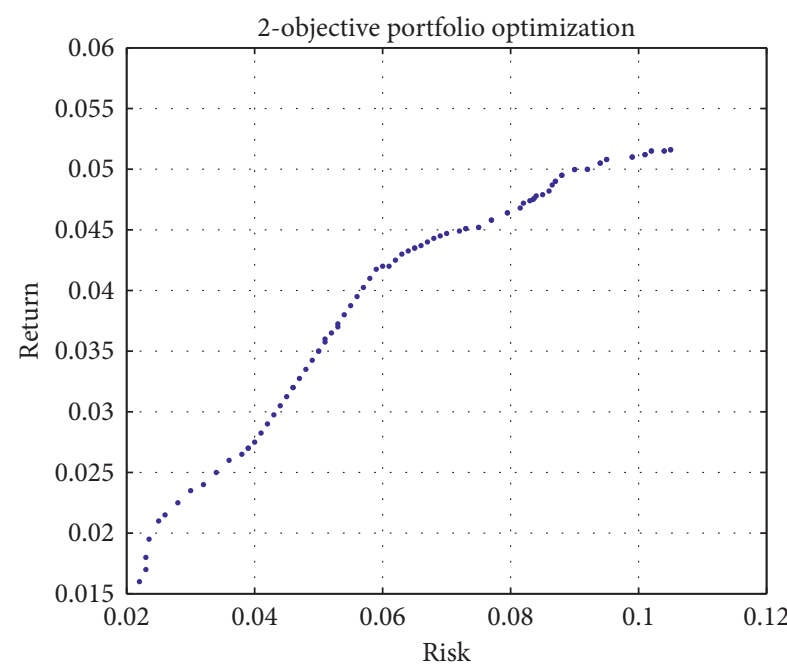

(b)

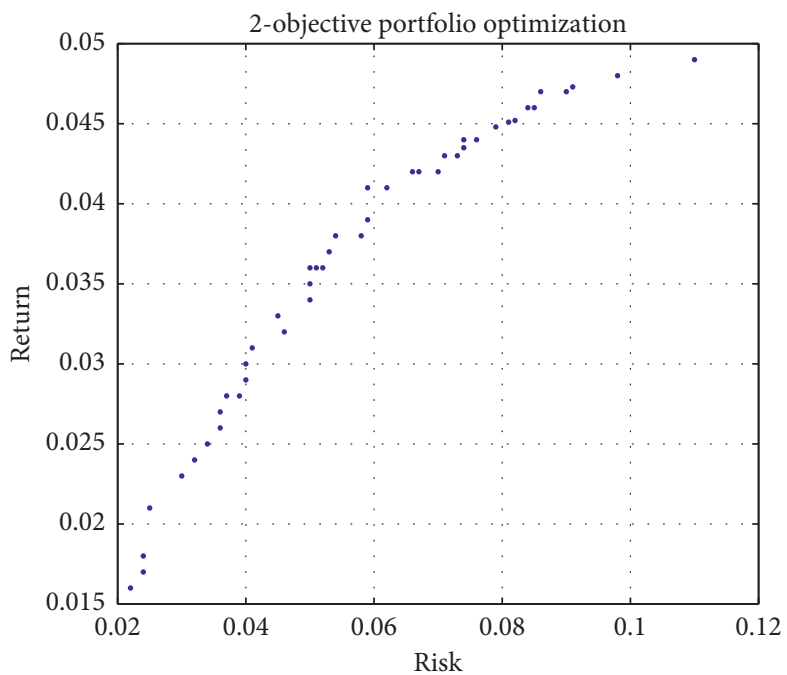

(c)

Figure 13: PF obtained by $p$-MORSGA and $p$-MOEAs on MV portfolio model: (a) PF obtained by $p$-MORSGA on MV portfolio model; (b) PF obtained by NSGA-II on MV portfolio model; (c) PF obtained by MOEA/D on MV portfolio model.

subject to $\sum_{i=1}^{N} w_{i}=1, \quad a_{i} z_{i} \leq w_{i} \leq b_{i} z_{i}, i=1,2, \ldots, N$

$$
0 \leq a_{i} \leq 1,0 \leq b_{i} \leq 1
$$

$z_{i}= \begin{cases}1, & \text { for } w_{i}>0 \\ 0, & \text { otherwise }\end{cases}$

$\sum_{i=1}^{N} z_{i}=K$

$\sum_{i j B}=\left(\frac{1}{T}\right) \sum_{i=1}^{T}\left[\operatorname{Min}\left(R_{i t}-B, 0\right) \cdot \operatorname{Min}\left(R_{j t}-B, 0\right)\right]$.
It should be noted that when appropriate indicators are available, the number of model objectives can also increase.

5.2. Applications for Portfolio Problem. The experiment uses daily historical data of 12 kinds of assets from Shanghai Stock Exchange, which are collected at the monthly rate of each stock from January 2010 to December 2016.

The frontier of $p$-MORSGA is searched and shown in Figures 13 and 14. In addition, since the true PF of the portfolio problem is not yet clear about p-MSMOEAs, all acquired PS are considered to be true PF [40]. The comparison results are listed in Tables 3 and 4, where hypervolume is used to test their performance.

Figure 13 shows the nondominant solution of the MV model generated by $p$-MORSGA. Investors can choose portfolio selection methods according to their risk preferences in order to obtain corresponding returns. 


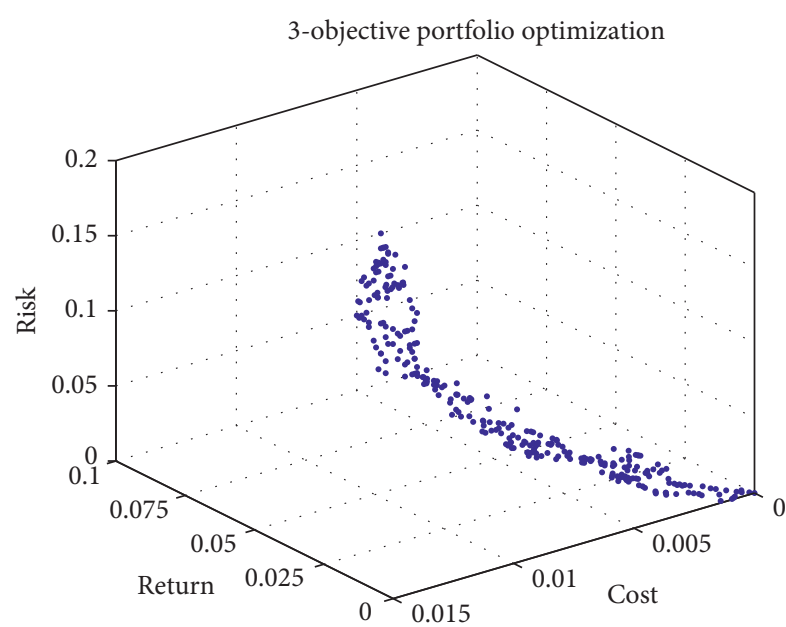

(a)

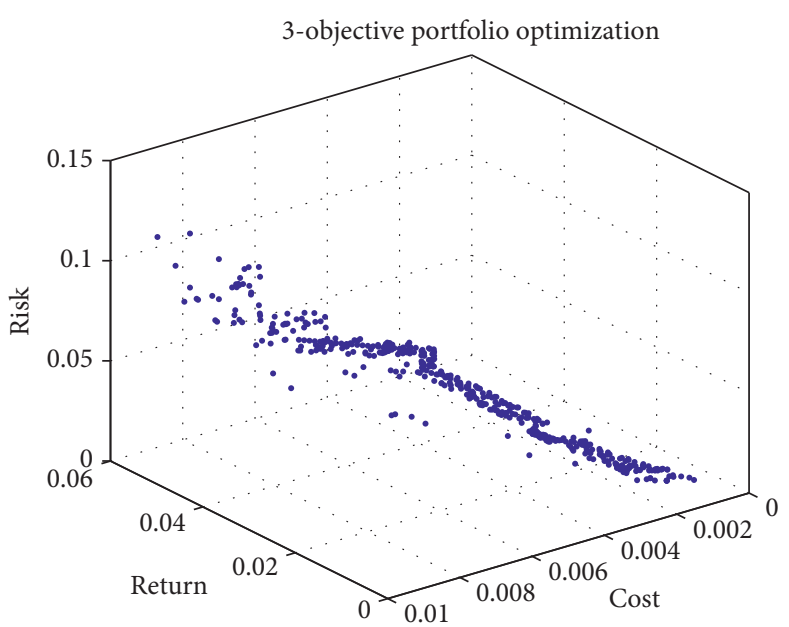

(b)

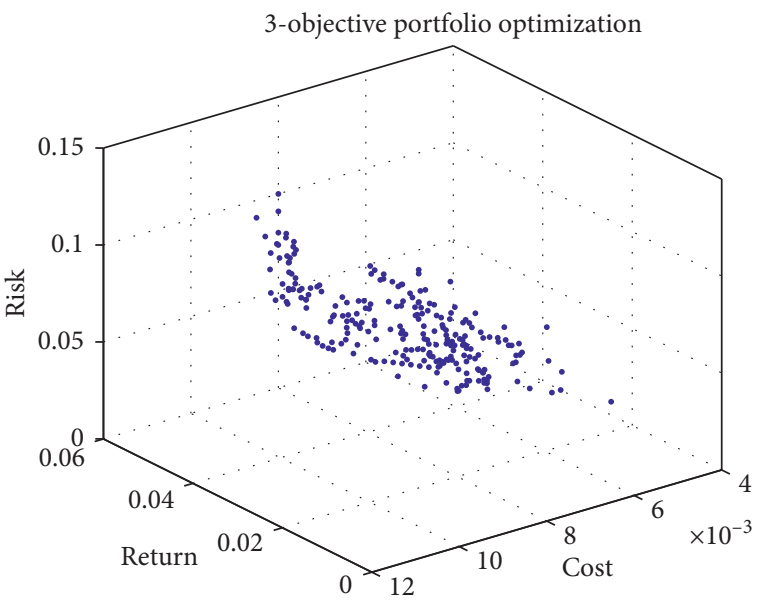

(c)

FIGURE 14: PF obtained by $p$-MORSGA and MOEAs on return-risk-cost portfolio model: (a) PF obtained by $p$-MORSGA on return-riskcost portfolio model; (b) PF obtained by NSGA-II on return-risk-cost portfolio model; (c) PF obtained by MOEA/D on return-risk-cost portfolio model.

TABLE 3: Comparison of performance on MV portfolio model.

\begin{tabular}{lcccr}
\hline MV portfolio model & & -MORSGA & NSGA-II & MOEA/D \\
\hline & Max & $\mathbf{1 . 1 3 e}-\mathbf{0 1}$ & $3.95 e-02$ & $3.44 e-02$ \\
Hypervolume indicator & Min & $\mathbf{4 . 2 0 e}-\mathbf{0 2}$ & $2.04 e-02$ & $9.93 e-03$ \\
& Avg & $\mathbf{8 . 7 4 e}-\mathbf{0 2}$ & $2.45 e-02$ & $1.57 e-02$ \\
& Std & $\mathbf{5 . 4 6 e}-\mathbf{0 1}$ & $4.77 e-02$ & $7.82 e-02$ \\
\hline
\end{tabular}

TABle 4: Comparison of performance on return-risk-cost portfolio model.

\begin{tabular}{llccr}
\hline Return-risk-cost portfolio model & & p-MORSGA & NSGA-II & MOEA/D \\
\hline & Max & $\mathbf{1 . 4 9 e + 0 0}$ & $5.41 e-01$ & $6.37 e-01$ \\
Hypervolume indicator & Min & $\mathbf{3 . 6 3 e}-\mathbf{0 1}$ & $9.86 e-02$ & $7.02 e-02$ \\
& Avg & $\mathbf{8 . 1 7 e}-\mathbf{0 1}$ & $1.07 e-01$ & $2.83 e-01$ \\
& Std & $\mathbf{3 . 9 5 e}-\mathbf{0 1}$ & $2.59 e-01$ & $3.91 e-01$ \\
\hline
\end{tabular}


TABle 5: Characteristics of the generation units.

\begin{tabular}{|c|c|c|c|c|c|c|}
\hline & G1 & G2 & G3 & G4 & G5 & G6 \\
\hline \multicolumn{7}{|c|}{ Generator limits } \\
\hline$P_{C \max }(\mathrm{MW})$ & 150 & 150 & 150 & 150 & 150 & 150 \\
\hline$P_{C \min }(\mathrm{MW})$ & 5 & 5 & 5 & 5 & 5 & 5 \\
\hline \multicolumn{7}{|c|}{ Cost coefficients } \\
\hline A & 10 & 10 & 20 & 10 & 20 & 10 \\
\hline B & 200 & 150 & 180 & 100 & 180 & 150 \\
\hline $\mathrm{C}$ & 100 & 120 & 40 & 60 & 40 & 100 \\
\hline \multicolumn{7}{|c|}{ Emission coefficients } \\
\hline A & 4.091 & 2.543 & 4.258 & 5.326 & 4.258 & 6.131 \\
\hline B & -5.554 & -6.407 & -5.094 & -3.550 & -5.094 & -5.555 \\
\hline$\Gamma$ & 6.490 & 5.638 & 4.586 & 3.380 & 4.586 & 5.151 \\
\hline$\Delta$ & $2.0 e-4$ & $5.0 e-4$ & $1.0 e-6$ & $2.0 e-3$ & $1.0 e-6$ & $1.0 e-5$ \\
\hline $\mathrm{E}$ & 2.857 & 3.333 & 8.000 & 2.000 & 8.000 & 6.667 \\
\hline
\end{tabular}

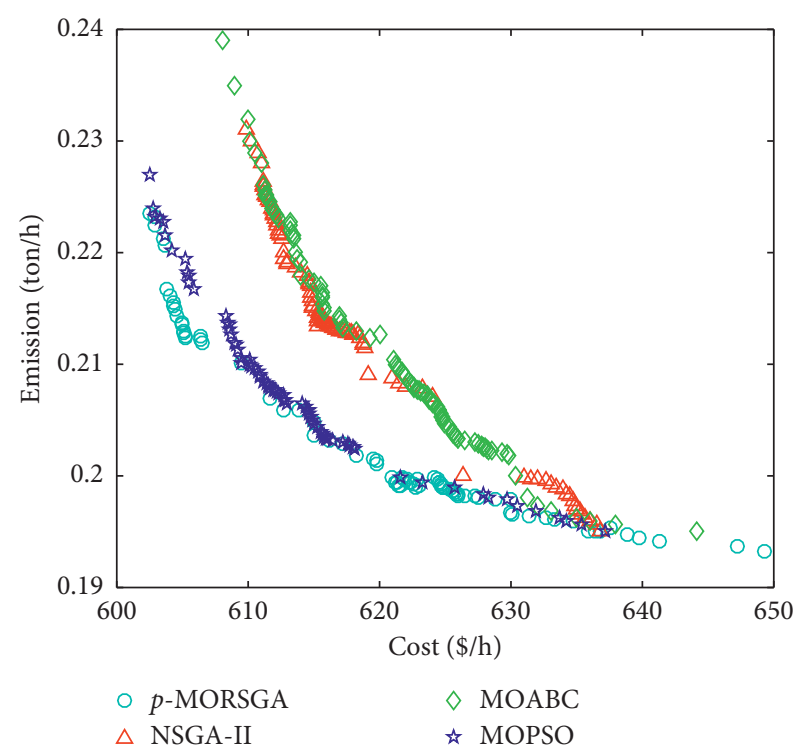

Figure 15: Pareto fronts obtained by $p$-MORSGA, MOPSO, MOABC, and NSGA-II for emission and cost.

In Figure 13, the effective curves of NSGA-II and the proposed algorithm obviously are continuous, but MODE locates the opposite. As can be seen from Table 3, the performance of $p$-MORSGA, NSGA-II, and MOEA/D in hypervolume index decreases in turn.

From Figure 14, when considering, risk and expected return are nearly contrary. The case of costs and risks, costs and expected benefits, regardless of another variable, is nearly the same. For facing all three variables, investors can choose portfolio selection methods according to their preferences.

As is visible from Figure 14, nondominant solutions' distribution in Figures 14(a) and 14(c) is more uniform and diversified. Obviously, as can be seen from Table 4, pMORSGA outperforms NSGA-II and MODE in terms of hypervolume indicators.

5.3. Optimal Power Flow Problem Formulation. OPF's main objective is to optimize restriction variables' setting while fulfilling several inequality constraints and equality. Generally speaking, OPF issues can be expressed mathematically as follows:

$$
\begin{array}{cl}
\text { minimize } & F(x, u)=\left(f_{1}(x, u), \ldots, f_{m}(x, u)\right), \\
\text { subject to } & g(x, u)=0, \\
& h(x, u) \leq 0,
\end{array}
$$

where $F$ represents the objective function, $g(x, u)$ is the equality constraint, and $h(x, u)$ is the system operation constraint. Here, $u$ is a vector of independent control variables, including

$$
x^{T}=\left[P_{\mathrm{G} 1}, V_{L 1}, \ldots, V_{L N_{\mathrm{pq}}}, Q_{\mathrm{G} 1}, \ldots, Q_{\mathrm{GN}}, S_{L 1}, \ldots, S_{L N_{1}}\right],
$$

where $P_{\mathrm{G} 1}$ is the active power output of the generator; $V_{L}$ is the load (PQ) bus voltage; $Q_{\mathrm{G}}$ is the reactive power output of the generator; $S_{L}$ is the transmission line load; $N_{\mathrm{pq}}$ denotes the number of PQ buses; and $N_{l}$ denotes the total number of transmission lines.

5.3.1. Objective Function. In this paper, there are three competing objective functions regarding the OPF problem, namely, total fuel cost, total power loss, and total emission cost, while satisfying several inequality constraints and equality.

The problem is usually expressed as follows.

(1) Minimize Total Fuel Costs. The curve is expressed as follows:

$$
f_{\mathrm{cos} t}=\sum_{i=1}^{N_{g}} f_{i}\left(a_{i} P_{\mathrm{G} i}^{2}+b_{i} P_{\mathrm{G} i}+c_{i}\right),
$$

where $a_{i}, b_{i}$, and $c_{i}$ are equivalent to the fuel cost coefficient of the $i$ th generator and $P_{\mathrm{G} i}$ is the actual power output of the ith generator.

(2) Minimization of Total Power Losses. Minimize total power loss as follows: 


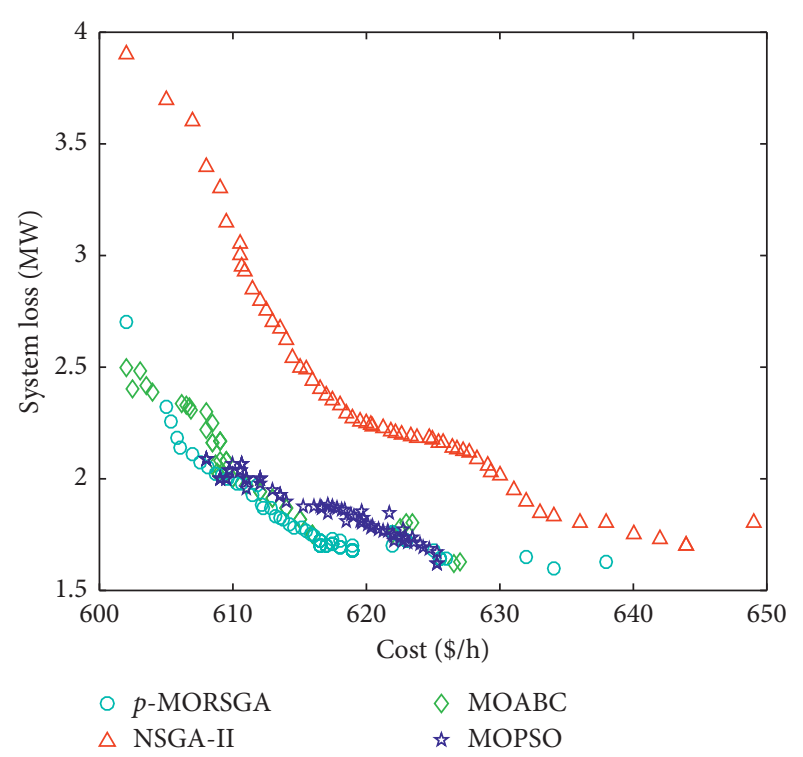

Figure 16: Pareto fronts obtained by p-MORSGA, MOPSO, MOABC, and NSGA-II for cost and loss.

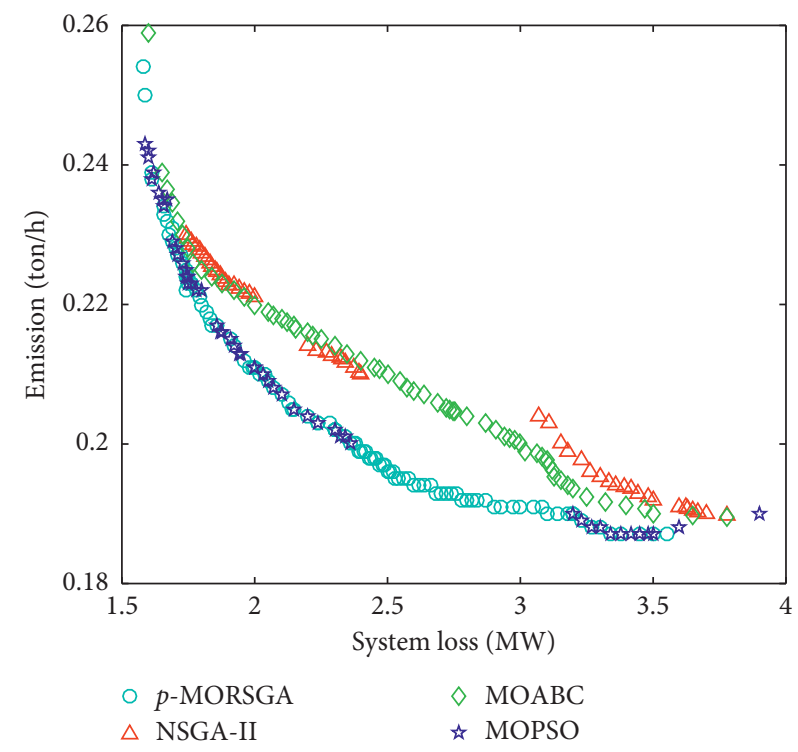

Figure 17: Pareto fronts obtained by $p$-MORSGA, MOPSO, MOABC, and NSGA-II for emission and loss.

$$
f_{\text {lost }}=\sum_{k=1}^{N_{l}} g_{k}\left(V_{i}^{2}+V_{j}^{2}-2 V_{i} V_{j} \cos \left(\delta_{i}-\delta_{j}\right)\right),
$$

where $N_{l}$ represents the number of transmission lines, $V$ expresses the voltage magnitudes, and $\delta$ represents the voltage angles.

(3) Minimization of Total Emission Cost. The total cost of emission is defined as [19]

$$
f_{\text {emission }}=\sum_{i=1}^{N_{g}}\left(\alpha_{i}+\beta_{i} P_{\mathrm{G} i}+\gamma_{i} P_{\mathrm{G} i}^{2}\right),
$$

where $f_{\text {emission }}$ represents total emission cost.
5.3.2. Equality Constraints. From (20), the equality constraint $g(x, U)$ is defined as follows:

$$
\begin{array}{r}
P_{G i}-P_{D i}-V_{i} \sum_{j=1}^{N} V_{j}\left(G_{i j} \cos \left(\delta_{i}-\delta_{j}\right)+B_{i j} \sin \left(\delta_{i}-\delta_{j}\right)\right)=0, \\
i=1,2, \ldots, N, \\
Q_{G i}-Q_{D i}-V_{i} \sum_{j=1}^{N} V_{j}\left(G_{i j} \cos \left(\delta_{i}-\delta_{j}\right)-B_{i j} \sin \left(\delta_{i}-\delta_{j}\right)\right)=0, \\
i=1,2, \ldots, N,
\end{array}
$$

where $P$ and $Q$ are the active and reactive power loads, respectively, and $G$ and $B$ are the real and imaginary parts.

5.3.3. Inequality Constraints. The inequality constraints are the power system handling.

(1) Generator Constraints. Generator active power PG, generator reactive power $\mathrm{QG}$, and generator voltage amplitude QG:

$$
\begin{array}{cl}
P_{G i, \min } \leq P_{G i} \leq P_{G i, \max }, & i=1, \ldots, N_{g}, \\
Q_{G i, \min } \leq Q_{G i} \leq Q_{G i, \max }, & i=1, \ldots, N_{g}, \\
V_{G i, \min } \leq V_{G i} \leq V_{G i, \max }, & i=1, \ldots, N_{g} .
\end{array}
$$

(2) Transformer Constraints. Transformer taps have restrictions as follows:

$$
T_{G i, \min } \leq T_{i} \leq T_{i, \max } \quad i=1, \ldots, N_{t} .
$$

(3) Switchable VAR Sources. The switchable VAR sources have minimum and maximum setting limits:

$$
Q_{C i, \min } \leq Q_{C i} \leq Q_{C i, \max } \quad i=1, \ldots, N_{c} .
$$

(4) Security Constraints. The limitations of load bus voltage amplitude and transmission line flow limitation are as follows:

$$
\begin{gathered}
V_{L i, \min } \leq V_{L i} \leq V_{L i, \max }, \quad i=1, \ldots, N_{p g} \\
\left|S_{L i}\right| \leq S_{L i, \max }, \quad i=1, \ldots, N_{l} .
\end{gathered}
$$

5.4. Multiobjective Optimal Power Flow Based on p-MORSGA. To verify the numerical correctness and the effectiveness of p-MORSGA, the multiobjective OPF problem will be used to implement simulation experiments. This algorithm will be applied to two objectives and three objectives of the OPF problem, and this problem is solved.

\subsubsection{Steps for $p$-MORSGA for OPF Problems}

Step 1. Input the system parameters and the minimum and maximum limits of control variables.

Step 2. Input parameters of $p$-MORSGA and the lower and upper limits of each variable. 
Table 6: The best compromise solutions for cost, emission, and loss.

\begin{tabular}{lcccc}
\hline & $p$-MORSGA & MOABC & NSGA-II & MOPSO \\
\hline PG1 & 18.9832 & 17.8933 & 35.6514 & 21.9132 \\
PG2 & 33.085 & 27.0342 & 53.9965 & 47.6396 \\
PG3 & 67.0249 & 70.8835 & 45.7462 & 90.2566 \\
PG4 & 82.1065 & 85.2331 & 55.0045 & 84.3363 \\
PG5 & 29.0455 & 27.0337 & 46.0104 & 7.0304 \\
PG6 & 53.2144 & 53.3102 & 622.449 & 65.4609. \\
f1 fuel cost & 610.0513 & 614.0154 & 0.2323 & 631.3809 \\
f2 (emission) & 0.2219 & 0.2376 & 3.9912 & 0.2448 \\
f3 (loss) & 2.1488 & 2.1790 & & 2.8491 \\
\hline
\end{tabular}

TABLE 7: The best solutions for cost and emission.

\begin{tabular}{|c|c|c|c|c|c|c|c|c|}
\hline & \multicolumn{2}{|c|}{$p$-MORSGA } & \multicolumn{2}{|c|}{ MOABC } & \multicolumn{2}{|c|}{ NSGA-II } & \multicolumn{2}{|c|}{ MOPSO } \\
\hline & Best f1 & Best f2 & Best f1 & Best $\mathrm{f} 2$ & Best $\mathrm{fl}$ & Best f2 & Best f1 & Best $\mathrm{f} 2$ \\
\hline PG1 & 11.66 & 40.03 & 10.72 & 7.37 & 34.82 & 41.67 & 25.88 & 55.67 \\
\hline PG2 & 30.23 & 47.52 & 28.51 & 41.98 & 54.88 & 47.64 & 31.88 & 45.82 \\
\hline PG3 & 54.33 & 55.82 & 55.75 & 60.73 & 50.03 & 49.79 & 61.84 & 55.22 \\
\hline PG4 & 103.63 & 42.04 & 104.83 & 100.88 & 45.02 & 42.99 & 100.83 & 39.98 \\
\hline PG5 & 42.62 & 51.28 & 43.99 & 41.51 & 54.72 & 51.80 & 44.88 & 54.30 \\
\hline PG6 & 37.94 & 53.62 & 36.50 & 34.99 & 43.88 & 51.60 & 37.98 & 47.99 \\
\hline f1 (cost) & 605.62 & 640.02 & 609.23 & 640.93 & 609.60 & 641.64 & 606.98 & 639.36 \\
\hline f2 (emission) & 0.2526 & 0.2031 & 0.2537 & 0.1998 & 0.2287 & 0.1898 & 0.2482 & 0.1937 \\
\hline
\end{tabular}

TABLE 8: The best solutions for cost and loss.

\begin{tabular}{lcccccccc}
\hline & \multicolumn{2}{c}{ p-MORSGA } & \multicolumn{2}{c}{ MOABC } & \multicolumn{2}{c}{ NSGA-II } & \multicolumn{2}{c}{ MOPSO } \\
& Best f1 & Best f3 & Best f1 & Best f3 & Best f1 & Best f3 & Best f1 & Best f3 \\
\hline PG1 & 14.99 & 3.99 & 9.26 & 4.02 & 18.98 & 3.58 & 67.00 \\
PG2 & 28.99 & 26.22 & 32.61 & 11.29 & 19.98 & 22.88 & 37.54 \\
PG3 & 54.79 & 106.00 & 58.03 & 108.99 & 39.22 & 71.94 & 67.26 & 10.68 \\
PG4 & 110.46 & 56.99 & 96.21 & 67.76 & 119.69 & 100.02 & 100.03 & 70.63 \\
PG5 & 30.21 & 5.01 & 45.83 & 11.87 & 41.12 & 5.20 & 36.54 \\
PG6 & 39.03 & 86.98 & 40.03 & 79.88 & 45.51 & 81.81 & 28.25 \\
f1 (cost) & 606.16 & 624.88 & 610.12 & 627.34 & 609.24 & 634.26 & 606.81 \\
f3 (loss) & 2.4554 & 1.5202 & 2.5021 & 1.5421 & 3.4197 & 1.6740 & 2.01 \\
\hline
\end{tabular}

Table 9: The best solutions for emission and loss.

\begin{tabular}{|c|c|c|c|c|c|c|c|c|}
\hline & \multicolumn{2}{|c|}{$p$-MORSGA } & \multicolumn{2}{|c|}{ MOABC } & \multicolumn{2}{|c|}{ NSGA-II } & \multicolumn{2}{|c|}{ MOPSO } \\
\hline & Best f2 & Best f3 & Best f2 & Best f3 & Best f2 & Best f3 & Best $\mathrm{f} 2$ & Best f3 \\
\hline PG1 & 44.92 & 9.00 & 33.60 & 11.98 & 43.11 & 62.48 & 32.04 & 11.01 \\
\hline PG2 & 45.22 & 4.98 & 58.98 & 6.23 & 40.42 & 142.98 & 45.34 & 76.78 \\
\hline PG3 & 55.66 & 98.45 & 50.53 & 69.54 & 56.33 & 106.22 & 60.99 & 22.87 \\
\hline PG4 & 40.92 & 69.65 & 33.52 & 94.12 & 39.99 & 61.55 & 42.99 & 62.80 \\
\hline PG5 & 47.02 & 4.47 & 61.33 & 6.28 & 40.49 & 4.99 & 48.78 & 81.78 \\
\hline PG6 & 53.25 & 102.62 & 47.00 & 94.99 & 35.00 & 35.80 & 51.45 & 59.99 \\
\hline f2 (emission) & 0.2003 & 0.2587 & 0.2004 & 0.3100 & 0.2398 & 0.2134 & 0.2061 & 0.2603 \\
\hline f3 (loss) & 4.1198 & 1.5935 & 4.7623 & 1.6345 & 4.3061 & 2.2314 & 4.1351 & 1.6501 \\
\hline
\end{tabular}


TABLE 10: The best compromise solutions for emission and cost.

\begin{tabular}{|c|c|c|c|c|}
\hline & $p$-MORSGA & MOABC & NSGA-II & MOPSO \\
\hline PG1 & 25.0102 & 24.8816 & 35.1988 & 24.3028 \\
\hline PG2 & 39.0058 & 38.4973 & 54.4014 & 36.7983 \\
\hline PG3 & 56.4611 & 38.1053 & 49.0943 & 54.3984 \\
\hline PG4 & 72.0211 & 74.8915 & 46.0004 & 71.9823 \\
\hline PG5 & 51.3025 & 46.1210 & 56.1235 & 51.8923 \\
\hline PG6 & 45.5280 & 41.8988 & 43.1914 & 44.04728 \\
\hline f1 $(\operatorname{cost})$ & 614.8302 & 615.5069 & 625.9913 & 615.2733 \\
\hline $\mathrm{f} 2$ (emission) & 0.1998 & 0.2000 & 0.1988 & 0.2014 \\
\hline
\end{tabular}

TABLE 11: The best compromise solutions for loss and cost.

\begin{tabular}{lcccc}
\hline & $p$-MORSGA & MOABC & NSGA-II & MOPSO \\
\hline PG1 & 4.3822 & 8.5941 & 3.5519 & 10.5911 \\
PG2 & 24.9932 & 28.1041 & 12.8737 & 87.8831 \\
PG3 & 78.9744 & 73.6835 & 92.0908 & 81.9942 \\
PG4 & 104.1988 & 104.6922 & 28.0134 & 95.3140 \\
PG5 & 8.9811 & 16.5047 & 62.7941 & 11.9914 \\
PG6 & 61.9852 & 49.8818 & 622.2812 & 71.4352 \\
f1 (cost) & 620.5899 & 622.2713 & 1.7991 & 628.2924 \\
f3 (loss) & 1.6791 & 1.6854 & & 1.5963 \\
\hline
\end{tabular}

TABLE 12: The best compromise solutions for loss and emission.

\begin{tabular}{lcccc}
\hline & p-MORSGA & MOABC & NSGA-II & MOPSO \\
\hline PG1 & 20.0945 & 67.7844 & 29.7010 & 22.6015 \\
PG2 & 29.9966 & 22.9971 & 32.2034 & 76.7044 \\
PG3 & 80.3288 & 29.7523 & 66.0093 & 102.9912 \\
PG4 & 65.1966 & 66.0088 & 34.1155 & 63.9801 \\
PG5 & 17.0043 & 76.4533 & 48.0114 & 6.5088 \\
PG6 & 71.2054 & 20.9199 & 2.6788 & 86.0299 \\
f2 (emission) & 1.8956 & 2.0087 & 0.2166 & 1.9204 \\
f3 (loss) & 0.1953 & 0.2078 & 0.2525 \\
\hline
\end{tabular}

Step 3. Input the minimum and maximum limits of variables.

Step 4. Produce the initial population.

Step 5. Cluster the population by $K$-means and compute the fitness.

Step 6. Store nondominated solutions in the memory table.

Step 7. Optimize every cluster individually.

Step 8. Handle the constraints.

Step 9. Compute the fitness of the solutions and store nondominated solutions into EA.

Step 10. Update each EA of each hive.

Step 11. Judge the condition of reclustering. If it meets the condition, the number of clusters can be reset and go to Step 4; if not, go to Step 7.

5.4.2. Simulation Results. The standard IEEE 30 bus system has been applied as a test system [23]. The system contains six generators, 41 transmission lines, and 4 transformers. The system data are shown in Table 5 [41].
In this section, we consider the following two test system scenarios:

(1) Two objectives: the emission cost, the emission loss, and the loss cost are considered separately.

(2) Three objectives: emissions, costs, and losses.

(1) Case I: Two-Objective OPF Optimization. The new algorithm is compared with three MOEAs in three aspects: minimum cost, minimum emission, and minimum loss. Figures 15-17 show the performance of four algorithms, respectively. Tables 10-12 show the two-objection compromise solution data. Finally, Table 7 shows the best solutions for minimum cost and emission. Table 8 provides data on emission losses and loss costs. Table 9 shows the best solutions for loss and emission.

First, two competing objectives need to be considered: fuel costs and emissions. From Figure 15, we can make out that the $p$-MORSGA method can provide a uniformly distributed solution. From Table 7 , it is clear that the minimum fuel cost that is obtained by $p$-MORSGA is $605.62 \$ / \mathrm{h}$, which is more wonderful than the results that are 


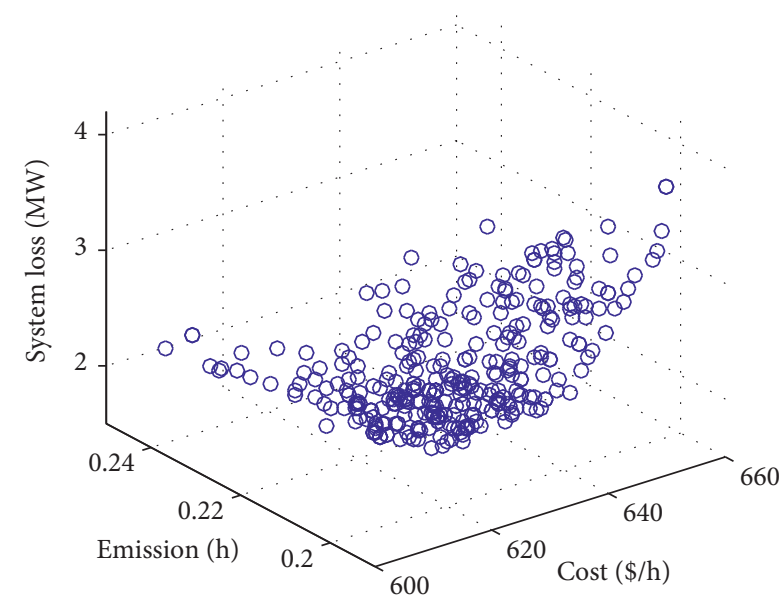

(a)

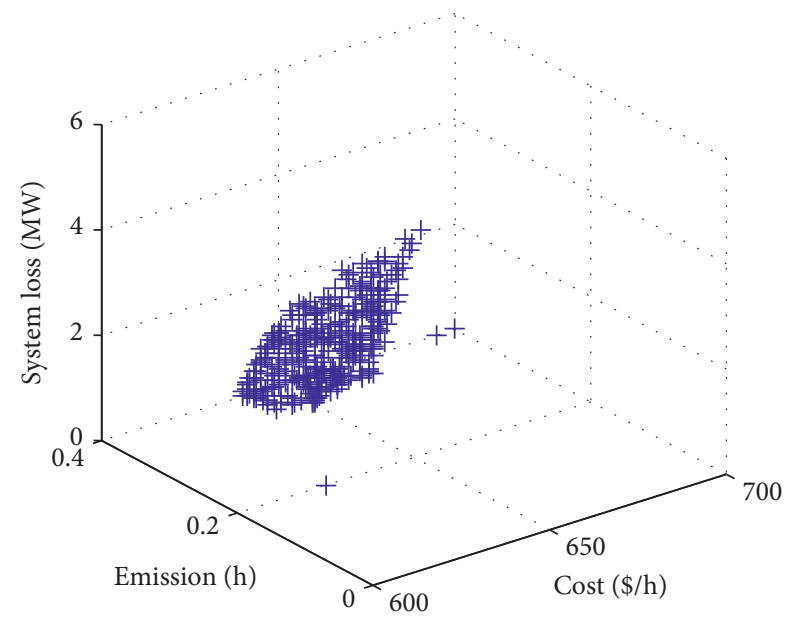

(c)

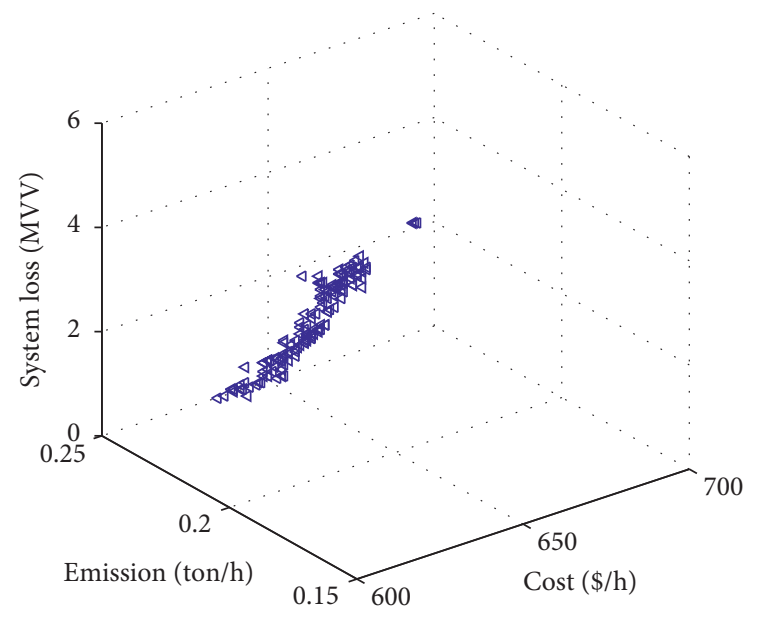

(b)

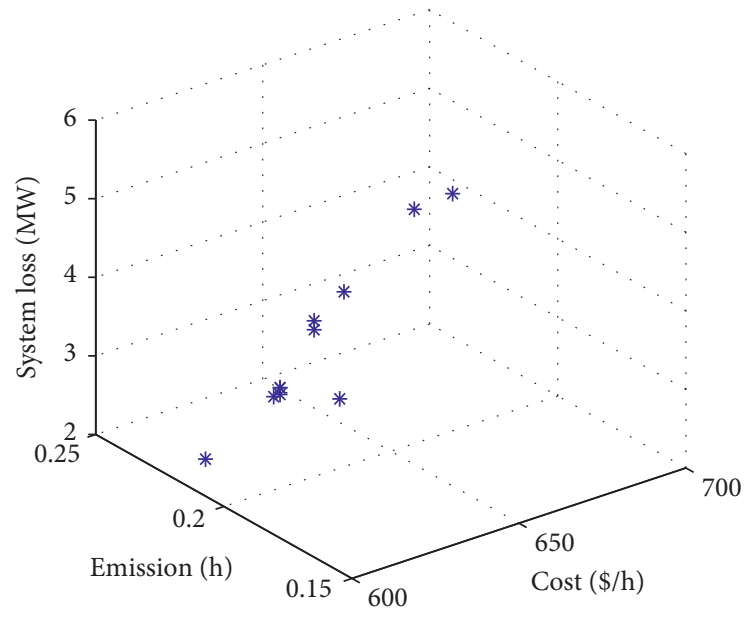

(d)

Figure 18: Pareto fronts obtained by CMOABC, MOPSO, MOABC, and NSGA-II for fuel cost, emission and loss: (a) p-MORSGA; (b) MOABC; (c) MOPSO; (d) NSGA-II.

TABLE 13: The best solutions for cost, emission, and loss.

\begin{tabular}{|c|c|c|c|c|c|c|c|c|c|c|c|c|}
\hline & \multicolumn{3}{|c|}{$p$-MORSGA } & \multicolumn{3}{|c|}{ MOABC } & \multicolumn{3}{|c|}{ MOPSO } & \multicolumn{3}{|c|}{ NSGA-II } \\
\hline & Best f1 & Best $\mathrm{f} 2$ & Best f3 & Best $\mathrm{f} 1$ & Best $\mathrm{f} 2$ & Best f3 & Best f1 & Best $\mathrm{f} 2$ & Best f3 & Best f1 & Best f2 & Best f3 \\
\hline PG1 & 20.20 & 43.99 & 5.88 & 34.88 & 3.18 & 17.95 & 21.92 & 37.02 & 8.99 & 24.99 & 36.89 & 17.95 \\
\hline PG2 & 20.56 & 45.77 & & & 15.47 & & & & & & & 26.43 \\
\hline PG3 & 69.52 & 60.02 & 104.25 & 61.37 & 80.41 & 60.66 & 64.99 & 62.55 & 81.38 & 56.88 & 49.54 & 93.88 \\
\hline PG4 & 100.76 & 39.10 & 69.00 & 40.55 & 68.99 & 100.09 & 100.61 & 46.77 & 62.39 & 47.29 & 38.88 & 65.2 \\
\hline PG5 & 45.99 & 53.89 & 4.98 & 43.99 & 9.06 & 37.77 & 39.29 & 50.12 & 66.45 & 44.55 & 50.99 & 8.07 \\
\hline PG6 & 36.55 & 47.13 & 86.44 & 35.76 & 77.02 & 40.99 & 33.66 & 52.34 & 57.77 & 41.88 & 49.21 & 67.99 \\
\hline f1 (cost) & 610.99 & 650.99 & 637.44 & 639.99 & 625.78 & 612.98 & 615.99 & 640.10 & 661.27 & 615.23 & 639.12 & 643.02 \\
\hline f2 (emission) & 0.2503 & 0.2065 & 0.2499 & 0.2154 & 0.2507 & 0.2610 & 0.3799 & 0.2388 & 0.4046 & 0.2453 & 0.1989 & 0.2599 \\
\hline f3 (loss) & 2.0012 & 4.1707 & 1.5944 & 4.0062 & 1.6931 & 2.0002 & 2.1074 & 2.4576 & 1.5010 & 2.4002 & 2.3422 & 2.1055 \\
\hline
\end{tabular}

optimized using the other three algorithms. And when the best fuel cost effect case is considered, the $p$-MORSGA method can provide smaller loss. The best compromise solutions are $614.8302 \$ / \mathrm{h}$ and $0.1998 \mathrm{t} / \mathrm{h}$ ( $p$-MORSGA), as shown in Table 10. Once again, testify that the method can get a better solution.

The objective function of cost loss is shown in Figure 16. We can see from Figure 16 that the proposed algorithm obtains better results than the other three algorithms.
From Table 9, it can be concluded that the $p$-MORSGA method can provide lower economic fuel cost when considering the minimum loss, while the $p$-MORSGA method can provide smaller loss when considering the optimal fuel cost effect.

For the emission loss objective function, we can come to know that the algorithm achieves performance ranking similar to that of the fuel cost emission objective function. 
(2) Case II: Tree-Objective OPF Optimization. In this instance, each algorithm needs to optimize three objectives. Figure 18 is the convergence curve and Table 13 is the list of data results. Compared with the four convergence effects of Figure 18, the Pareto optimal front solutions obtained by $p$ MORSGA are uniformly distributed and widely distributed, while the results obtained by MOABC in the other three algorithms are better.

Judging from the data in Table 13, for $p$-MORSGA, when $\mathrm{F} 1$ (cost) is $610.99 \$ / \mathrm{h}, \mathrm{F} 2$ (emission) is 0.2503 ton $/ \mathrm{h}$, and F3 (loss) is $2.0021 \mathrm{MW}, \mathrm{F} 1$ is greater than $610.99 \$ / \mathrm{h}$ for the other three algorithms and the other two objectives are relatively poor when $\mathrm{F} 1$ is the smallest. It can be concluded that $p$-MORSGA can obtain better solutions than other algorithms on this three-objective problem.

Referring to the data in Table 6 , the proposed $p$ MORSGA obtains the best pollution emission value and generation cost. Comparatively speaking, $p$-MORSGA has the best optimization effect.

\section{Conclusion}

In this paper, a multiobjective optimizer based on $p$-optimality and global general (GG) is proposed, which is called p-MORSGA. Through eight mathematical benchmark functions, $p$-MORSGA has an outstanding performance on accuracy and convergence. It can be inferred that $p$ MORSGA is simple in structure, easy to implement, and has great potential to solve complex MOP. In order to prove its effectiveness in solving multiobjective realistic problems, the performance of $p$-MORSGA is tested by using multiobjective combinatorial optimization problem (MOPOP) and multiobjective optimal power flow (OPF) problems. The MOPOP includes three objectives: expected return, risk, and expected cost. The experimental results show that the proposed $p$-MORSGA is considered to be able to obtain the distribution of multiple solutions. For OPF problem, a 30 bus IEEE test system is used to test the proposed algorithm. Compared with three other OPF problems with different objectives, the proposed method can provide uniformly distributed Pareto optimal solutions.

\section{Data Availability}

The MATLAB data used to support the findings of this study are available from the corresponding author upon request.

\section{Conflicts of Interest}

The authors declare that they have no conflicts of interest.

\section{Acknowledgments}

This work was supported by the National Key Research and Development Program of China under Grant No. 2017YFB1103603 and 2017YFB1103003, National Natural Science Foundation of China under Grant No. 61602343, 51607122, 61772365, 41772123, 61802280, 61806143, 51575158, and 61502318, Tianjin Province Science and
Technology Projects under Grant No. 17JCYBJC15100 and 17JCQNJC04500, and Basic Scientific Research Business Funded Projects of Tianjin (2017KJ093 and 2017KJ094).

\section{References}

[1] A. Babalik, A. Ozkis, S. A. Uymaz, and M. S. Kiran, "A multiobjective artificial algae algorithm," Applied Soft Computing, vol. 68 , pp. $377-395,2018$.

[2] M. Hamdy, A.-T. Nguyen, and J. L. M. Hensen, "A performance comparison of multi-objective optimization algorithms for solving nearly-zero-energy-building design problems," Energy and Buildings, vol. 121, no. 6, pp. 57-71, 2016.

[3] J. Wang, P. Du, T. Niu, and W. Yang, "A novel hybrid system based on a new proposed algorithm-multi-objective whale optimization algorithm for wind speed forecasting," Applied Energy, vol. 208, pp. 344-360, 2017.

[4] Y. Liu, L. Fan, Y. Wang, and Q. Dong, "Optimization of logistics nodes in dynamic location with a multi-objective evolutionary algorithm," in Proceedings of the 2009 International Conference on Computational Intelligence \& Security IEEE Computer Society, IEEE, Beijing, China, December 2009.

[5] D. K. Lim, D. K. Woo, H. K. Yeo, S. Y. Jung, J. S. Ro, and H. K. Jung, "A novel surrogate-assisted multi-objective optimization algorithm for an electromagnetic machine design," IEEE Transactions on Magnetics, vol. 51, no. 3, pp. 1-4, 2015.

[6] N. Garćia-Pedrajas, C. Hervás-Martínez, and J. Muñoz-Pérez, "Multi-objective cooperative coevolution of artificial neural networks (multi-objective cooperative networks)," Neural Networks, vol. 15, no. 10, pp. 1259-1278, 2002.

[7] K. Deb, A. Pratap, S. Agarwal, and T. Meyarivan, "A fast and elitist multiobjective genetic algorithm: NSGA-II," IEEE Transactions on Evolutionary Computation, vol. 6, no. 2, pp. 182-197, 2002.

[8] E. Zitzler and S. Künzli, "Indicator-based selection in multiobjective search," in Lecture Notes in Computer Science, pp. 832-842, 2004.

[9] Q. Zhang and H. Li, "MOEA/D: a multiobjective evolutionary algorithm based on decomposition," IEEE Transactions on Evolutionary Computation, vol. 11, no. 6, pp. 712-731, 2007.

[10] H. Zhang, Y. Zhu, and H. Chen, "Root growth model: a novel approach to numerical function optimization and simulation of plant root system," Soft Computing, vol. 18, no. 3, pp. 521-537, 2014.

[11] S. W. Kembel and J. F. Cahill Jr., "Plant phenotypic plasticity belowground: a phylogenetic perspective on root foraging trade-offs," The American Naturalist, vol. 166, no. 2, pp. 216-230, 2005.

[12] S. W. Kembel, H. De Kroon, J. F. Cahill Jr., and L. Mommer, "Improving the scale and precision of hypotheses to explain root foraging ability," Annals of Botany, vol. 101, no. 9, pp. 1295-1301, 2008.

[13] A. Hodge, "Root decisions," Plant, Cell \& Environment, vol. 32, no. 6, pp. 628-640, 2009.

[14] K. Deb, "Multi-objective genetic algorithms: problem difficulties and construction of test problems," Evolutionary Computation, vol. 7, no. 3, pp. 205-230, 1999.

[15] S. Agrawal, Y. Dashora, M. Tiwari, and Y. J. Son, "Interactive particle swarm: a pareto-adaptive metaheuristic to multiobjective optimization," IEEE Transactions on Systems, Man, and Cybernetics-Part A: Systems and Humans, vol. 38, no. 2, pp. 258-277, 2008. 
[16] M. Laumanns, L. Thiele, K. Deb, and E. Zitzler, "Combining convergence and diversity in evolutionary multiobjective optimization," Evolutionary Computation, vol. 10, no. 3, pp. 263-282, 2002.

[17] L. Ben Said, S. Bechikh, and K. Ghedira, "The r-dominance: a new dominance relation for interactive evolutionary multicriteria decision making," IEEE Transactions on Evolutionary Computation, vol. 14, no. 5, pp. 801-818, 2010.

[18] S. Yang, M. Li, X. Liu, and J. Zheng, "A grid-based evolutionary algorithm for many-objective optimization," IEEE Transactions on Evolutionary Computation, vol. 17, no. 5, pp. 721-736, 2013.

[19] F. Goulart and F. Campelo, "Preference-guided evolutionary algorithms for many-objective optimization," Information Sciences, vol. 329, pp. 236-255, 2016.

[20] L. Thiele, K. Miettinen, P. J. Korhonen, and J. Molina, "A preference-based evolutionary algorithm for multi-objective optimization," Evolutionary Computation, vol. 17, no. 3, pp. 411-436, 2009.

[21] K. Mohanty, T. Mitra, H. Saxén, and N. Chakraborti, "Multiple criteria in a top gas recycling blast furnace optimized through ak-optimality-based genetic algorithm," Steel Research International, vol. 87, no. 10, pp. 1284-1294, 2016.

[22] G. Eslami and M. Z. Kabir, "Multi-objective optimization of orthogonally stiffened cylindrical shells using optimality criteria method," Scientia Iranica, vol. 22, no. 3, pp. 717-727, 2015.

[23] P. J. Bentley and J. P. Wakefield, "Finding acceptable solutions in the pareto-optimal range using multiobjective genetic algorithms," in Soft Computing in Engineering Design and Manufacturing, pp. 231-240, Springer, Berlin, Germany, 1998.

[24] F. di Pierro, S.-T. Khu, and D. A. Savi, "An investigation on preference order ranking scheme for multiobjective evolutionary optimization," IEEE Transactions on Evolutionary Computation, vol. 11, no. 1, pp. 17-45, 2007.

[25] L. Li, W. Wang, and X. Xu, "Multi-objective particle swarm optimization based on global margin ranking," Information Sciences, vol. 375, pp. 30-47, 2017.

[26] A. R. Pouya, M. Solimanpur, and M. J. Rezaee, "Solving multiobjective portfolio optimization problem using invasive weed optimization," Swarm \& Evolutionary Computation, vol. 28, pp. 42-57, 2016.

[27] M. K. Mehlawat and P. Gupta, "Fuzzy chance-constrained multiobjective portfolio selection model," IEEE Transactions on Fuzzy Systems, vol. 22, no. 3, pp. 653-671, 2014.

[28] M. Jiang, Z. Meng, and C. Dang, "A multiobjective conditional value-at-risk model in time interval for loan portfolios," International Journal of Management Science and Engineering Management, vol. 3, no. 4, pp. 253-265, 2008.

[29] X. Yuan, B. Zhang, P. Wang et al., "Multi-objective optimal power flow based on improved strength pareto evolutionary algorithm," Energy, vol. 122, pp. 70-82, 2017.

[30] H. Pulluri, R. Naresh, and V. Sharma, "An enhanced selfadaptive differential evolution based solution methodology for multiobjective optimal power flow," Applied Soft Computing, vol. 54, pp. 229-245, 2017.

[31] K. Teeparthi and D. M. Vinod Kumar, "Multi-objective hybrid PSO-APO algorithm based security constrained optimal power flow with wind and thermal generators," Engineering Science and Technology, an International Journal, vol. 20, no. 2, pp. 411-426, 2017.

[32] E. C. Jara, "Multi-objective optimization by using evolutionary algorithms: the $\$ \mathrm{p} \$$-optimality criteria," IEEE
Transactions on Evolutionary Computation, vol. 18, no. 2, pp. 167-179, 2014.

[33] Y. Hu, H. Chen, M. He, L. Sun, R. Liu, and H. Shen, "Multiswarm multi-objective optimizer based on $\mathrm{p}$-optimality criteria for multi-objective portfolio management," Mathematical Problems in Engineering, vol. 2019, Article ID 8418369, 22 pages, 2019.

[34] W. Zhang and J. L. Zhang, "Improved NSGA-II algorithm based on differential evolution mechanism," in Proceedings of the 2017 36th Chinese Control Conference, (CCC '17), pp. 4334-4338, IEEE, Dalian, China, July 2017.

[35] F. Xue, A. C. Sanderson, and R. J. Graves, "Pareto-based multiobjective differential evolution," in Proceedings of the 2003 Congress on Evolutionary Computation (CEC '03), vol. 2, pp. 862-869, IEEE, Canberra, Australia, December 2003.

[36] H. Ishibuchi, Y. Sakane, N. Tsukamoto, and Y. Nojima, "Evolutionary many-objective optimization by NSGA-II and MOEA/D with large populations," in Proceedings of the 2009 IEEE International Conference on Systems, Man and Cybernetics, pp. 1820-1825, IEEE, San Antonio, TX, USA, October 2009.

[37] M. Elarbi, S. Bechikh, A. Gupta, L. Ben Said, and Y.-S. Ong, "A new decomposition-based NSGA-II for many-objective optimization," IEEE Transactions on Systems, Man, and Cybernetics: Systems, vol. 48, no. 7, pp. 1191-1210, 2018.

[38] H. Markowitz, "Portfolio selection," The Journal of Finance, vol. 7, no. 1, pp. 77-91, 1952.

[39] S. Babaei, M. M. Sepehri, and E. Babaei, "Multi-objective portfolio optimization considering the dependence structure of asset returns," European Journal of Operational Research, vol. 244, no. 2, pp. 525-539, 2015.

[40] F. Cesarone, J. Moretti, and F. Tardella, "Optimally chosen small portfolios are better than large ones," Economics Bulletin, vol. 36, no. 4, pp. 1876-1891, 2016.

[41] Z.-H. Zhang, C.-Q. Zhong, Z.-Z. Xu, and H.-F. Teng, “A nondominated sorting cooperative Co-evolutionary differential evolution algorithm for multi-objective layout optimization," IEEE Access, vol. 5, pp. 14468-14477, 2017. 


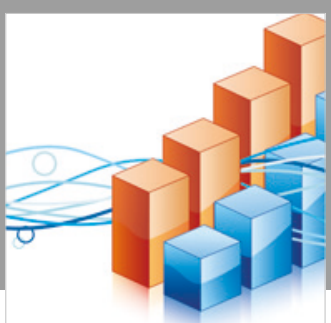

Advances in

Operations Research

\section{-n-m}
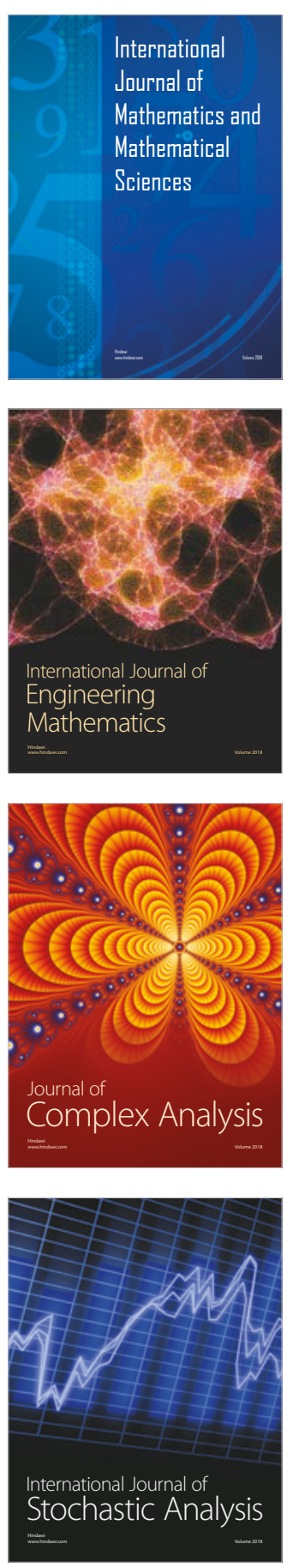
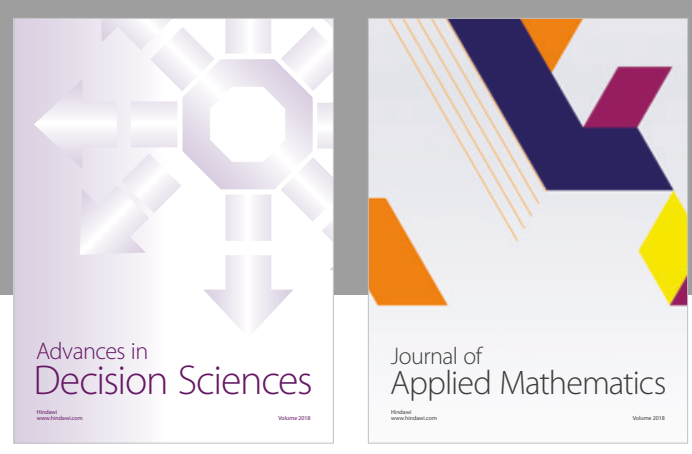

Journal of

Applied Mathematics
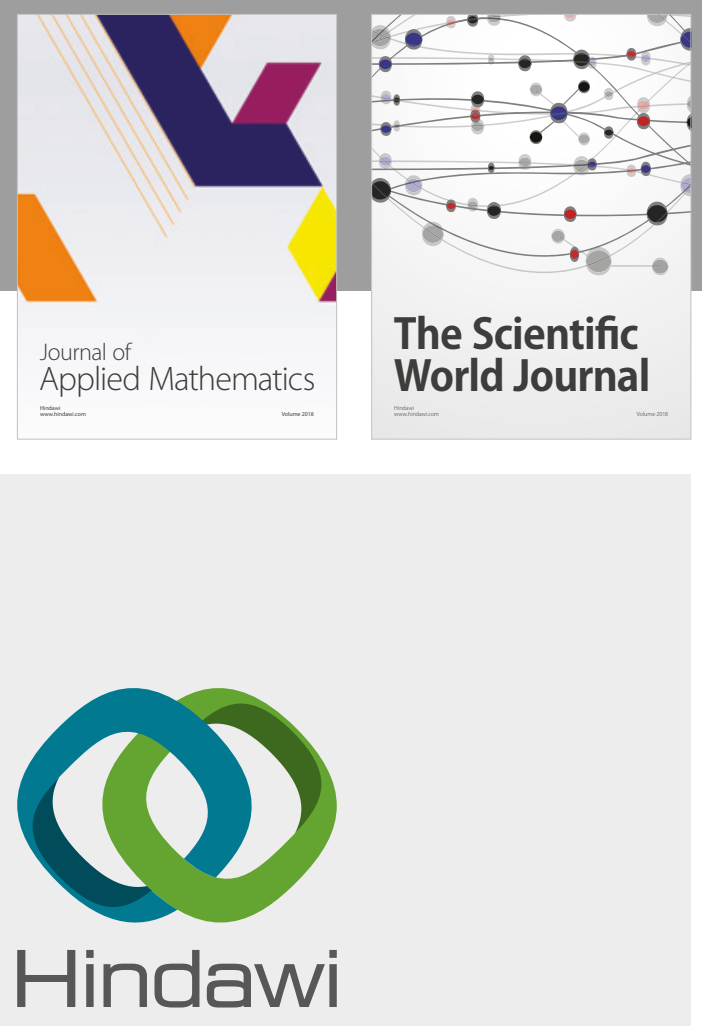

Submit your manuscripts at

www.hindawi.com

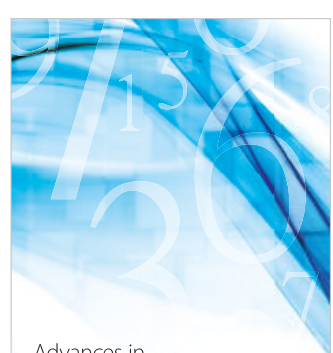

Advances in
Numerical Analysis
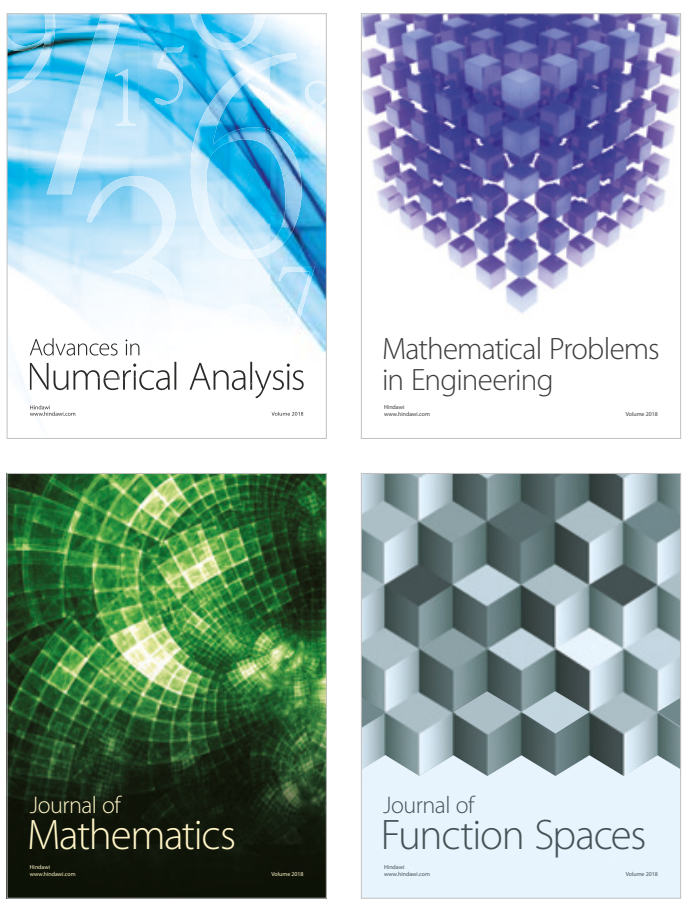

Mathematical Problems in Engineering

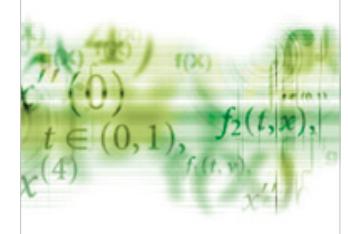

International Journal of

Differential Equations

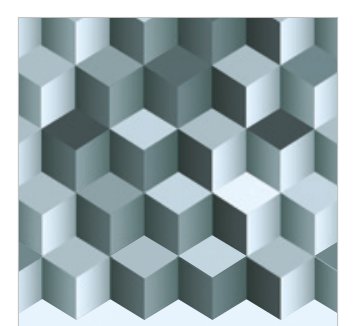

Journal of

Function Spaces

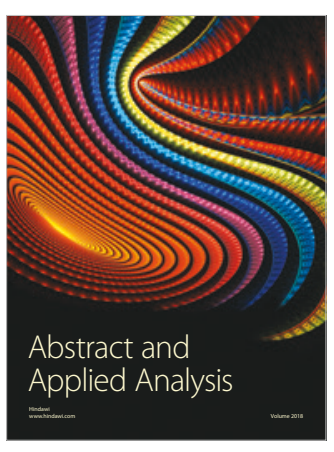

The Scientific

World Journal

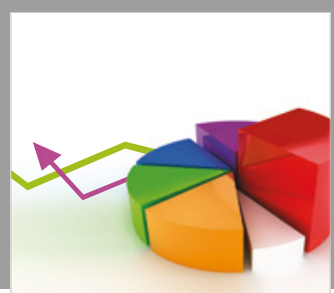

Journal of

Probability and Statistics
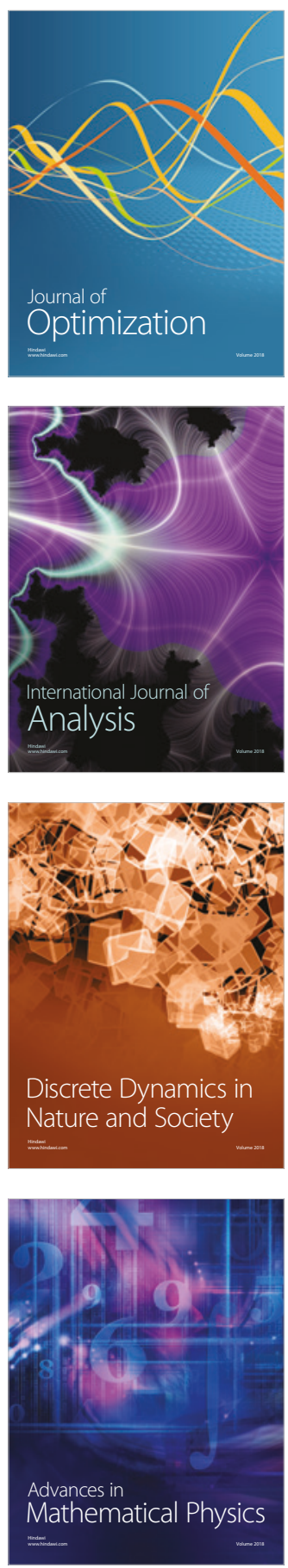